\title{
A Reevaluation of the Host Range and Geographical Distribution of Claviceps Species in the United States
}

\author{
Stephen C. Alderman, USDA-ARS National Forage Seed Production Research Center, 3450 SW Campus Way, \\ Corvallis, OR 97331; Richard R. Halse, Department of Botany and Plant Pathology, Oregon State University, Cor- \\ vallis, OR 97331; and James F. White, Department of Plant Pathology, Cook College - Rutgers University, New \\ Brunswick, NJ 80901
}

\begin{abstract}
Alderman, S. C., Halse, R. R., and White, J. F. 2004. A reevaluation of the host range and geographical distribution of Claviceps species in the United States. Plant Dis. 88:63-81.

A listing of host and state reports and distribution maps for 11 taxa of Claviceps occurring in the United States, including C. africana, C. cinerea, C. grohii, C. nigricans, C. paspali, C. pusilla, C. purpurea var. purpurea and var. spartinae, C. tripsaci, C. yanagawensis, and C. zizaniae, was prepared based on literature citations and examination of specimens from herbaria. The occurrence of $C$. ranunculoides is questioned based on examination of conidia and sclerotia from archived specimens. Collections of $C$. purpurea var. purpurea from grasses in the Panicoideae were referred to other Claviceps spp. based on occurrence of macroconidia and microconidia. C. purpurea var. purpurea was found on 165 grass species within the continental United States and Alaska. The size of conidia of $C$. purpurea var. purpurea was found to be relatively stable across host and geographical regions. However, conidia of $C$. purpurea var. purpurea from hosts in the Aveneae and Meliceae (generally associated with wet habitats) were more variable in size and generally larger than those from other tribes in the Pooideae. Claviceps spp. in the continental United States occurred in diverse habitats, including temperate grasslands of the middle to northern latitudes ( $C$. purpurea var. purpurea, $C$. nigricans) to the middle to southern latitudes (C. pusilla), coastal habitats (C. purpurea var. spartinae, C. zizaniae), northern wetlands (C. grohii), southern temperate to subtropical grasslands ( $C$. africana, C. paspali, C. tripsaci, C. yanagawensis), and arid southwestern grasslands (C. cinerea).
\end{abstract}

Additional keywords: disease assessment, disease distribution, ergot, host-pathogen index

The genus Claviceps includes species adapted to a variety of niches in habitats ranging from marine to desert and subtropical to arctic. Most species of Claviceps have a host range limited to a genus or several genera of grasses. The exception is Claviceps purpurea var. purpurea (Fr.:Fr.) Tul., a common pathogen of temperate grasses in the Pooideae. The host range of $C$. purpurea var. purpurea is reported to include up to 200 species in the United States (40,136). Langdon (96), in his 1954 study of Claviceps, recognized 25 species. Since then, 15 additional species have been described (3). Hosts reported susceptible to Claviceps spp. were summarized by Brady (18) and Kawatani (88).

Corresponding author: Steve Alderman

E-mail: aldermas@onid.orst.edu

Accepted for publication 21 August 2003.

Publication no. D-2003-1105-01S

This article is in the public domain and not copyrightable. It may be freely reprinted with customary crediting of the source. The American Phytopathological Society, 2004.
However, the occurrence of Claviceps spp. and their host and geographical distribution within the United States is not clearly defined.

Claviceps spp. are floral pathogens that infect the ovaries of susceptible hosts. Each infected ovary is replaced by a single sclerotium. During infection, macroand/or microconidia (and in some cases also secondary conidia), depending on the Claviceps sp., are produced. Conidia are responsible for secondary spread of Claviceps. The sclerotium and the disease are commonly referred to as ergot. Sclerotia of many of the Claviceps spp. contain toxic alkaloids, and their presence in feed or in pasture grasses can cause poisoning in animals. Sclerotia of Claviceps spp. can be collected with seed during harvest. $C$. purpurea var. purpurea, in particular, is a generally known contaminant of harvested cereal grains and grass seed. There is potential for introducing new Claviceps spp. into the United States with seed, although the impact of such an introduction would vary depending on the Claviceps sp. imported and the hosts affected. Of significance is the recent introduction of C. afri- cana, which is now an important problem in U.S. sorghum production (11). Recognition of new or emerging Claviceps spp. in the United States requires an understanding of the geographical and host distribution of Claviceps spp. currently in the country.

Claviceps species are differentiated by morphological features of the ascostroma, including color, distribution of perithecia within the stroma, dimensions of perithecia, asci, and ascospores, characteristics of the stipe, sclerotial and conidial morphology $(3,96)$, or by molecular genetic differences (120). Unfortunately, ascostromata are rarely observed in nature, and most collections include only ungerminated sclerotia. Nevertheless, morphology of sclerotia and conidia can be helpful in differentiating species of Claviceps. Sclerotial forms include the black to purpleblack, elongated sclerotia of $C$. purpurea; the roughly textured, spherical, tan to brown sclerotia of $C$. paspali F. Stevens \& J.G. Hall; the large, horse-tooth shaped, gray-white sclerotia of $C$. gigantea S.F. Fuentes, Ullstrup, \& Rodriquez; the greenish sclerotia of $C$. viridis Padwick \& Azmatullah; and the oblong, purple sclerotia of $C$. zizaniae (Fyles) Panidou (3). Although morphology of sclerotia may be helpful in differentiating some species of Claviceps, sclerotia of other species, e.g., C. digitariae Hansford, C. nigricans Tul., $C$. pusilla Cesati, and $C$. ranunculoides Möller, are similar in appearance to $C$. purpurea. Characteristics such as size and shape of macroconidia, microconidia, and secondary conidia are morphological features that can be used to further delimit Claviceps spp. (100). C. pusilla, for example, has characteristic triangular conidia (96) which appear to be unique among Claviceps spp. Host range also may be helpful in Claviceps spp. recognition. Species such as $C$. grohii J.W. Groves, $C$. nigricans, and $C$. zizaniae are believed to be restricted to a single host genus. Thus, recognition of many Claviceps species, in the absence of ascostromata, may be possible based on combined characteristics of conidia, sclerotia, and host range.

Although the concept of varieties of $C$. purpurea has not been widely accepted, 
recent arguments for a Claviceps sp. found on Spartina as a distinct variety of $C$. purpurea, $C$. purpurea var. spartinae, based on morphological, physiological, ecological, and host range differences (34), are convincing. Because of its wide host range, $C$. purpurea var. purpurea is often assumed to be a causal agent of Claviceps infection. For example, Androgon spp. are listed as hosts of $C$. purpurea var. purpurea $(23,61,97,125,129)$, presumably based on occurrence and appearance of sclerotia. However, when sclerotia from Andropogon spp. in Iowa were germinated (157), the ascostromata resembled $C$. pusilla and not C. purpurea var. purpurea. In another case, C. zizaniae was listed as $C$. purpurea var. purpurea until studies of Pantidou (117) established it as a different species. Further investigations may differentiate additional species of Claviceps from pathogens now identified as $C$. purpurea var. purpurea.

Listings of grass hosts for Claviceps spp. are complicated by the many recent changes in grass nomenclature. Differences among grass taxonomists concerning the synonymy of species can create confusion in citations. For example, Farr et al. (40) included the grass species Agrostis alba L. and $A$. palustris Huds. as synonyms of $A$. stolonifera L. Kartesz (87), on the other hand, listed $A$. alba as a synonym of $A$. gigantea Roth.

Studies of speciation, host range, and geographical distribution in the genus Claviceps require details of the host, site where the collection was made, and location of any corresponding voucher specimens. Most of the records of Claviceps were established from plant disease surveys during the 1930s through 1950s. For many of these records, specimens were sent to the herbarium of the USDA National Fungus Collection. However, collections in other herbaria may represent additional sources for host and geographical information for fungi in the genus Claviceps.

The objectives of this study were to: (i) compile an updated listing of grass species reported as natural hosts of Claviceps spp. based on published reports, inclusion in the USDA National Fungus Collection, or examination of herbarium collections in which hosts could be identified; (ii) summarize the listing with updated grass synonymies; (iii) map the distribution of Claviceps species in the United States with respect to host occurrence; (iv) evaluate the validity of conidia size as an attribute for comparing Claviceps spp.; and (v) identify questionable $C$. purpurea-host associations.

\section{MATERIALS AND METHODS}

An extensive literature search for reports of ergot in the United States was conducted. Reports in which species of Claviceps were not defined or host identities were uncertain were excluded. Cita- tions of ergot on the cereal grains, especially wheat and rye, are numerous, and citations included in this report serve primarily as examples for state occurrence. $C$. purpurea var. purpurea is generally recognized as occurring throughout the United States on wheat and rye $(9,136)$. Reports of C. microcephala (Wallr.) Tul. were included under $C$. purpurea var. purpurea since the species are considered synonymous $(3,96)$.

Only original citations, where possible, were included in the host-species listing. Curators of U.S. herbaria listed in Index Herbariorum (81) were consulted for information on records of Claviceps in their collections. Herbaria from which collections were obtained included: Herbarium, Botany Department, Field Museum of Natural History, Chicago, IL [F]; Farlow Herbarium of Cryptogamic Botany, Harvard University, Cambridge, MA $[\mathrm{FH}]$; Herbarium, University of Florida, Gainesville [FLAS]; Herbarium, U.S. National Fungus Collection, Beltsville, MD [BPI]; Herbarium, Center for Biodiversity, Illinois Natural History Survey, Champaign, IL [ILLS]; Ada Hayden Herbarium, Botany Department, Iowa State University, Ames [ISC]; Herbarium, University of Michigan, Ann Arbor [MICH]; Herbarium, Botany and Plant Pathology Department, East Lansing, MI [MSC]; Herbarium, Biological Sciences Division, University of Montana, Missoula [MONTU]; Bessey Herbarium, University of Nebraska State Museum, Lincoln [NEB]; Herbarium, New York Botanical Garden Herbarium, New York [NY]; Herbarium, Botany and Plant Pathology Department, Corvallis, OR [OSC]; Rocky Mountain Herbarium, University of Wyoming, Laramie [RM]; University Herbarium, University of California, Berkeley [UC]; Herbarium, Botany Department, University of Wisconsin, Madison [WIS]; and Herbarium, Plant Pathology Department, Washington State University, Pullman [WSP]. Letters in brackets correspond to herbarium codes listed in Index Herbariorum (81). Except for BPI, records from herbaria were included in host listings and state reports only after specimens were examined and verified for presence of Claviceps and verification of host identities. Collections that included only sclerotia were excluded. Data were summarized as the occurrence of host-Claviceps combinations within each state. The number of duplicate records at any given location was not tallied. New collections were deposited at herbarium OSC.

Conidia were examined from specimens obtained from herbaria or collections made during the course of this study. Collections of Claviceps spp. occurring outside the United States were also obtained and examined. Conidia were gently washed from individual sclerotia by placing the sclerotium in a small drop of water on a glass slide for about 30 to $60 \mathrm{~s}$. Conidia were examined at $\times 1,000$, and tracings of the first 25 conidia encountered were made with a drawing tube attached to an Olympus microscope. The taxonomic placement of grass genera into subfamilies and tribes was based on Watson and Dallwitz (161). Current nomenclature of grass genera/species followed that presented by Kartesz (87)

\section{RESULTS}

C. africana was reported from $\mathrm{GA}, \mathrm{KS}$, MS, and TX as a pathogen of Sorghum bicolor (L.) Moench and Sorghum halepense (L.) Pers. (Table 1, Fig. 1). Macroconidia were ellipsoidal-oblong, $14 \times 5 \mu \mathrm{m}$ (Table 2). Microconidia were spherical, about $3 \mu \mathrm{m}$ in diameter (Table 2).

Claviceps cinerea was reported from $\mathrm{AZ}, \mathrm{OK}, \mathrm{NM}$, and TX as a pathogen of Hilaria spp. (Table 1, Fig. 1). Macroconidia of $C$. cinerea were fusiform, 20-25 $\times$ 4-5 $\mu \mathrm{m}$. Microconidia were ellipsoidal, 6-8 $\times 0.5-0.7 \mu \mathrm{m}$ (Table 2).

C. grohii was reported on Carex spp. in MI, OR, and WA (Table 1, Fig. 1). Conidia of C. grohii were ellipsoidal-oblong, 11-16 $\times$ 4-5 $\mu \mathrm{m}$ (Table 2).

Claviceps junci was listed by Greene (61) as occurring in WI on Juncus nodosus (Table 1). $C$. junci is an invalid name because it is based on only the conidial stage. Conidia from collections of Claviceps on Juncus spp. from CA and Canada were similar in size and shape, ellipsoidaloblong, 12-13 $\times 4 \mu \mathrm{m}$ (Table 2).

C. nigricans occurred on Eleocharis spp. primarily in the Midwest, but its distribution extended to SC and CA (Table 1, Fig. 1). Conidia of $C$. nigricans from collections of Eleocharis spp. from CA were 9$11 \times$ about $4 \mu \mathrm{m}$ (Table 2 ).

C. paspali was distributed from MD to FL, along the Gulf States, and across to California (Fig. 1). C. paspali was confined to the genera Paspalum and Axonopus (Table 1). Conidia were ellipsoidal, $12 \times 4-5 \mu \mathrm{m}$ (Table 2).

Most hosts for $C$. purpurea var. purpurea occurred in the subfamily Pooideae. Mean conidial lengths for specimens in the Aveneae and Meliceae were more variable and generally larger than those from hosts in the Bromeae, Poeae, or Triticeae, where conidia were 5.5 to $7.9 \times 2.5$ to $3.9 \mu \mathrm{m}$ (Table 2). Most hosts of $C$. purpurea var. purpurea occurred in the northern half of the United States, although $C$. purpurea var. purpurea has been reported from all states in the continental United States. One hundred fifty-nine species were identified as susceptible to $C$. purpurea var. purpurea (Table 3), excluding host reports from panicoid grasses. The greatest host species diversity occurred in the midwestern and northwestern states (Fig. 2). More than 50 species of grasses were reported susceptible to $C$. purpurea var. purpurea in IA, $\mathrm{ND}$, and WA. Relatively few (10 or fewer) 
Table 1. List of plants reported as susceptible to Claviceps species, other than C. purpurea var. purpurea, among U.S. states, based on published reports or specimens in herbaria ${ }^{a}$

\begin{tabular}{|c|c|}
\hline Host & Source \\
\hline \multicolumn{2}{|l|}{ Bambusoideae } \\
\hline \multicolumn{2}{|l|}{ Diarheneae } \\
\hline \multicolumn{2}{|l|}{ Claviceps sp. } \\
\hline $\begin{array}{l}\text { Diarrhena americana Beauv. } \\
=\text { Diarrhena diandra (Michx.) A.W. Wood }\end{array}$ & KS (MIN); MO (BPI) \\
\hline \multicolumn{2}{|l|}{ Oryzeae } \\
\hline \multicolumn{2}{|l|}{ Claviceps zizaniae } \\
\hline Zizania aquatica $\mathrm{L}$. & $\begin{array}{l}\text { IA (53, 136); ME (BPI, NY, 136); MD (136); MN (91, 136); MO (BPI, 9); NE (136); ND } \\
\text { (BPI, NY, WSP; 136, 162); WA(BPI); WI }(61,136,159) ;\end{array}$ \\
\hline $\begin{array}{l}\text { Zizania palustris L. var. palustris } \\
=\text { Zizania aquatica var. angustifolia Hitchc. }\end{array}$ & IA (9); ME (BPI, FH, 9); MD (9); MN (OSC, 9, 127); MO (9); NE (9); ND (9); WI (9) \\
\hline \multicolumn{2}{|l|}{ Chloridoideae } \\
\hline \multicolumn{2}{|l|}{ Chlorideae } \\
\hline \multicolumn{2}{|l|}{ Claviceps cinerea } \\
\hline Hilaria belangeri var. longifolia (Vasey) A.S. Hitchc. & AZ (9); TX (BPI) \\
\hline Hilaria cenchroides Kunth & AZ $(67,136)$ \\
\hline Hilaria jamesii (Torr.) Benth. & NM (BPI) \\
\hline Hilaria mutica (Buckl.) Benth. & AZ (BPI, NY, WSP, 9, 67, 136); NM (BPI, 9, 99, 136); OK (9, 41, 126, 136); TX (BPI, FH, 9) \\
\hline \multicolumn{2}{|l|}{ Claviceps purpurea var spartinae ${ }^{\mathrm{b}}$} \\
\hline Spartina alterniflora Loisel & AL (36); CT (BPI); DE (BPI, NY, 9, 36, 136); FL (36, 4); GA (BPI, 36); LA (BPI, 9, 36, \\
\hline = Spartina stricta (Ait.) Roth. var. glabra (Muhl.) Gray & 136); ME (BPI, WIS, 9, 136); MD (BPI, 36); MA (BPI, MSC, 9, 36, 69, 136); MS (BPI, \\
\hline $\begin{array}{l}=\text { Spartina alterniflora var. pilosa } \text { (Merr.) Fern. } \\
=\text { Spartina glabra } \text { Muhl. }\end{array}$ & $\begin{array}{l}\text { 9, 35, 36, 110, 118, 136); NH (36); NJ (BPI, 9, 34, 36, 136); NY (BPI, 9, 136); NC (BPI, } \\
36,49,54) ; \mathrm{RI}(36,47,48) ; \mathrm{SC}(\mathrm{OSC}, 36) ; \mathrm{VA}(36)\end{array}$ \\
\hline Spartina cynosuroides (L.) Roth & FL (BPI, 9); IA (115); MS (36); NE (BPI); NY $(9,136)$ \\
\hline Spartina foliosa Trin. & CA (BPI, F, MSC, WIS); VA (BPI) \\
\hline $\begin{array}{l}\text { Spartina maritima (M.A. Curtis) Fern. } \\
=\text { Spartina stricta (Aiton) Roth }\end{array}$ & CA (BPI); LA (BPI); ME (BPI); MA (BPI); \\
\hline Spartina patens (Ait.) Muhl. & ME (BPI); MD (BPI, 9, 136); MA (BPI, FH); MS (36); NY (NY); NC (54); TX (BPI) \\
\hline $\begin{array}{l}\text { Spartina pectinata Link } \\
=\text { Spartina michauxiana Hitchc. }\end{array}$ & $\begin{array}{l}\text { CT (BPI); IA (53, 136); MN (127); MT (BPI , OSC, NY, 9, 136); NE (NEB, 136); OK (9, } \\
\text { 41, 136); SD (BPI, 9, 106, 136) }\end{array}$ \\
\hline \multicolumn{2}{|l|}{ Claviceps yanagawensis ${ }^{\mathrm{b}}$} \\
\hline Zoysia japonica Steudal & FL (4); VA (BPI) \\
\hline $\begin{array}{l}\text { Zoysia matrella }(\mathrm{L} .) \text { Merrill } \\
=\text { Zoysia pungens Willd. }\end{array}$ & $\mathrm{DC}(\mathrm{BPI})$ \\
\hline
\end{tabular}

Panicoideae

Andropogoneae

Claviceps africana

Sorghum bicolor (L.) Moench

Sorghum halepense (L.) Pers.

Claviceps pusilla

Andropogon gerardii Vitman

= Andropogon furcatus Muhl. in Wild.

Andropogon hallii Hack.

Bothriochloa ischaemum (L.) Keng

= Andropogon ischaemum $\mathrm{L}$.

Schizachyrium scoparium (Michx.) Nash

= Andropogon scoparius Michx.

Claviceps sp. ${ }^{\mathrm{b}}$

Andropogon gerardii Vitman

$=$ A. furcatus Muhl. in Willd.

Andropogon hallii Hack.

Andropogon virginicus $\mathrm{L}$.

Bothriochloa ischaemum L. var. ischaemum

= Andropogon ischaemum $\mathrm{L}$.

Bothriochloa saccharoides (Sw.) Rydb.

= Andropogon saccharoides $\mathrm{Sw}$.

= Amphilophis torreyanus (Steudall) Nash

Ischaemum muticum $\mathrm{L}$.

= Andropogon repens

Saccharum alopecuroides (L.) Nutt.

= Erianthus alopecuroides (L.) Ell.

Schizachyrium scoparium (Michx.) Nash

= Andropogon scoparius Michx.

Schizachyrium tenerum Nees

= Andropogon tener (Nees) Kunth

Sorghastrum nutans (L.) Nash

Spodiopogon sibiricus Trin.

Themeda forskalii Hackel ex Duthie

GA (160); KS (160); MS (166); TX (OSC, 83, 160)

GA (160); KS (160); TX $(82,160)$

IA (ICS, 52); NM (BPI)

IA (ICS, 52); OK (BPI)

IA (116); TX (BPI)

IA (52)

GA (9); KS (BPI, NY, 9); NE (129); NM (BPI); OK (BPI, 97, 125, 136); TX (BPI, 9, 23,

136); WI $(61,136)$

NE (129); OK (BPI, 41, 97, 125, 136); TX $(23,136)$

FL (9); MS (9); OK (9)

TX (BPI)

TX (BPI, 9, 30)

$\mathrm{OH}(121)$

AL (136); OK $(9,126,136)$

OK (BPI, 125, 136)

OK (41)

IA (ICS); KS (BPI, 9, 136); OK (9, 41, 125, 97, 136); TX (BPI, 9, 23, 136)

$\mathrm{DC}(\mathrm{BPI})$

$\mathrm{DC}(\mathrm{BPI})$

(Continued on next page)

\footnotetext{
a Herbaria were inventoried for ergot during 1996 to 1998. Herbarium codes are those listed in Index Herbariorum (81).

b Some reports list $C$. purpurea or $C$. sp.
} 
Table 1. (Continued from preceding page)

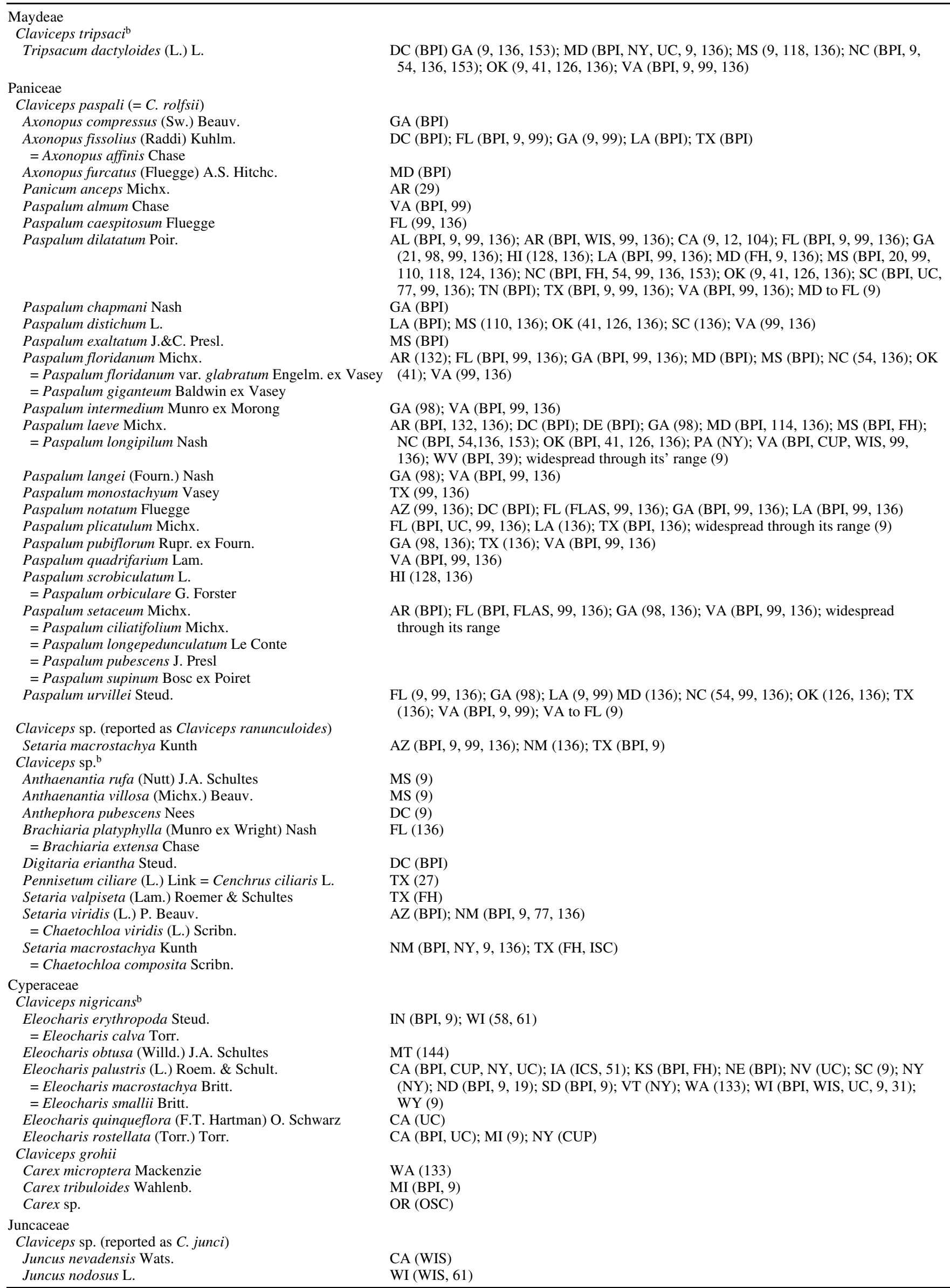


species were associated with $C$. purpurea var. purpurea in the southern states and the Atlantic coast states. Nearly all hosts susceptible to $C$. purpurea var. purpurea occurred in the subfamily Pooideae, with some exceptions in the Arundinoideae (Table 3). The report of $C$. purpurea var. purpurea on the bambusoid grass Diarrhena is questionable. Sclerotia from Diarrhena were slightly flattened but triangular in cross section, with collections brown to blue-black or gray to blue-black. C. purpurea var. purpurea was also considered doubtful on panicoid host genera, including Andropogon, Bothriochloa, Brachiaria, Digitaria, Saccharum, Schizachyrium, Setaria, Sorgastrum, and Spodiopogon based on the presence of macroconidia and microconidia. Collections from Andropogon, Digitaria, Setaria, and Sorghastrum were observed with microconidia and macroconidia (Table 2).

Claviceps purpurea var. spartinae occurred on Spartina spp. within the range of growth of Spartina along the Atlantic, Pacific, and Gulf Coast states and the Midwest. Conidia were ellipsoidal, oblong, or allantoid, 10.2 to $12.7 \mu \mathrm{m}$.

C. pusilla was reported from IA, OK, NM, and TX. Hosts included Andropogon

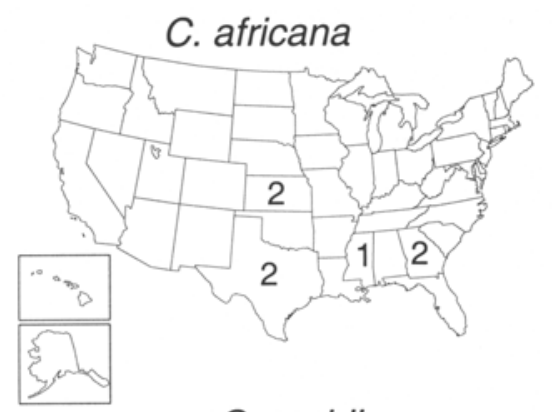

C. grohii

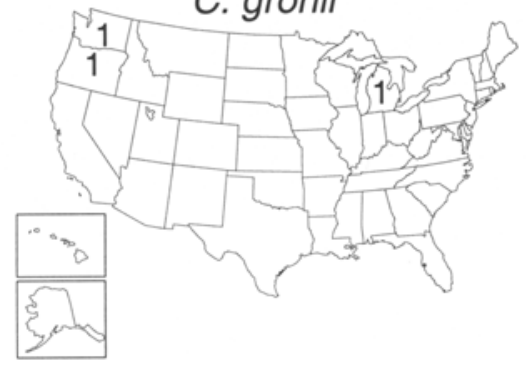

C. paspali

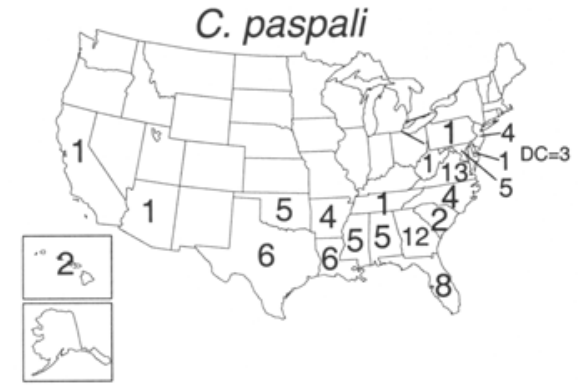

C. purpurea var. spartinae

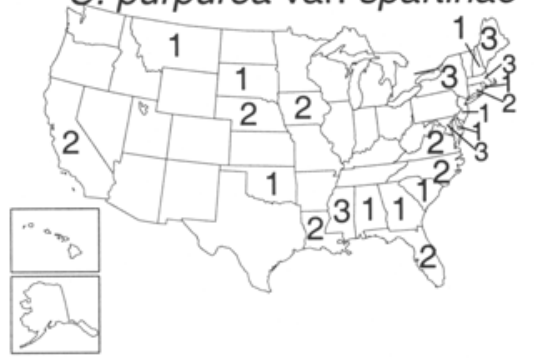

C. yanagawensis

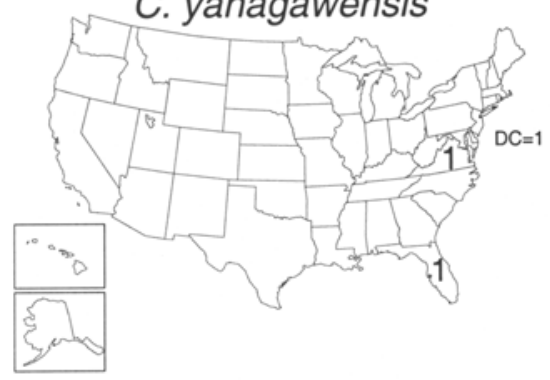

C. cinerea

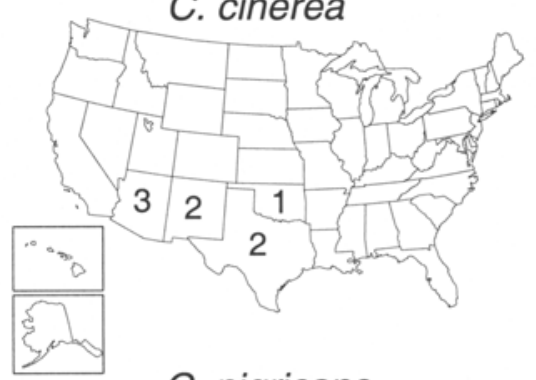

C. nigricans

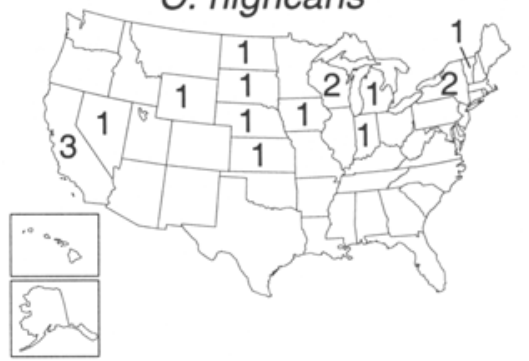

C. pusilla

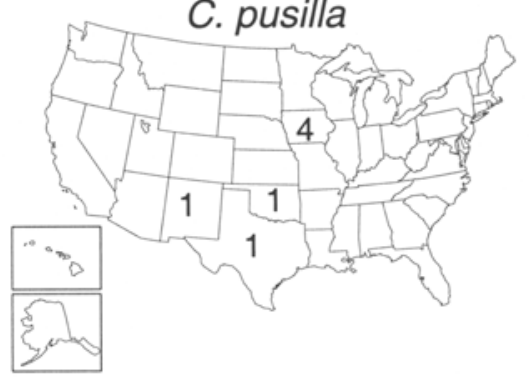

C. tripsaci

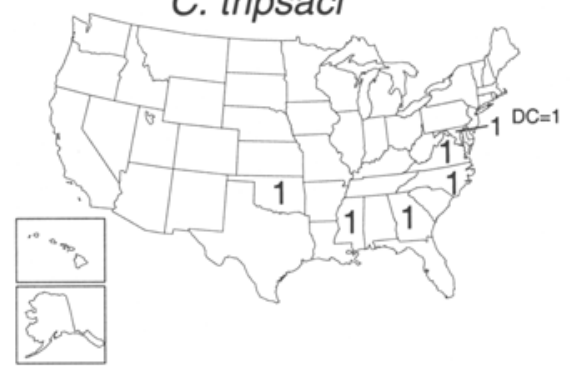

C. zizaniae

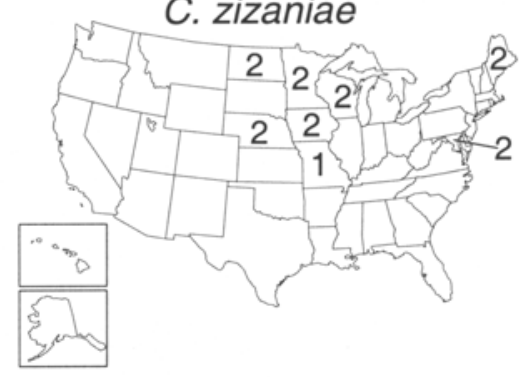

Fig. 1. For each Claviceps species, the number of grass species on which the particular Claviceps species was reported for each state is recorded. 
Table 2. Conidial attributes of Claviceps species within subfamilies and tribes of grasses (Poaceae), sedges (Cyperaceae) and rushes (Juncaceae)

\begin{tabular}{|c|c|c|c|c|c|c|}
\hline Species & Location & $\begin{array}{c}\text { Conidial } \\
\text { type }\end{array}$ & $\begin{array}{c}\text { Conidial } \\
\text { length }(\mu \mathrm{m})\end{array}$ & $\begin{array}{c}\text { Conidial } \\
\text { width }(\mu \mathrm{m})\end{array}$ & Conidial shape & Source \\
\hline \multicolumn{7}{|l|}{ Arundinoideae } \\
\hline \multicolumn{7}{|l|}{ Danthonieae } \\
\hline \multicolumn{7}{|l|}{ Claviceps sp. } \\
\hline Danthonia spicata & WI & & $9.5 \pm 1.4$ & $4.2 \pm 0.5$ & Ellipsoidal-oblong & BPI 633839 \\
\hline \multicolumn{7}{|l|}{ Stipeae } \\
\hline \multicolumn{7}{|l|}{ Claviceps purpurea } \\
\hline Achnatherum lettermanii & WY & & $5.8 \pm 0.8$ & $3.1 \pm 0.4$ & Ellipsoidal & RM 26100 (labeled Stipa lettermanii) \\
\hline Achnatherum nelsonii & UT & & $5.8 \pm 0.9$ & $3.2 \pm 0.4$ & Ellipsoidal & BPI 634439 (labeled Stipa columbiana) \\
\hline Achnatherum scribneri & $\mathrm{CO}$ & & $7.1 \pm 0.9$ & $3.2 \pm 0.4$ & Ellipsoidal & $\begin{array}{l}\text { NY: S. Boulder canyon, } 7,000 \mathrm{ft}, 1912 \text {, } \\
\text { Ex Herb. Arthur (labled Stipa scribneri) }\end{array}$ \\
\hline Nasella viridula & & & $7.2 \pm 1.2$ & $3.3 \pm 0.4$ & Ellipsoidal & ISC 318665 (labeled Stipa viridula) \\
\hline Oryzopsis asperfolia & NY & & $5.7 \pm 0.6$ & $2.7 \pm 0.3$ & Ellipsoidal & CUP 17762 \\
\hline Piptatherum miliaceum & $\begin{array}{c}\text { DC } \\
\text { (Australia) }\end{array}$ & & $8.1 \pm 0.9$ & $3.5 \pm 0.5$ & Ellipsoidal-oblong & BPI 633193 (labeled Oryzopsis milacea) \\
\hline \multicolumn{7}{|l|}{ Bambusoideae } \\
\hline \multicolumn{7}{|l|}{ Diarrheneae } \\
\hline \multicolumn{7}{|l|}{ Claviceps purpurea? } \\
\hline Diarrhena americana & $\mathrm{KS}$ & & $6.0 \pm 1.1$ & $2.4 \pm 0.8$ & Ellipsoidal-oblong & MIN 615560 \\
\hline Diarrhena americana & MO & & $5.7 \pm 0.8$ & $2.5 \pm 0.4$ & Ellipsoidal-oblong & BPI 633842 (labeled D. diandra) \\
\hline Diarrhena americana & MO & & $5.5 \pm 0.8$ & $2.3 \pm 0.3$ & Ellipsoidal-oblong & BPI 633841 (labeled D. diandra) \\
\hline \multicolumn{7}{|l|}{ Oryzeae } \\
\hline \multicolumn{7}{|l|}{ Claviceps zizanea } \\
\hline Zizanea aquatica & ND & & $11.2 \pm 1.6$ & $4.2 \pm 0.5$ & Ellipsoidal-oblong & $\begin{array}{l}\text { NY: near Fargo, 1900, Brenckle Fungi } \\
\text { Dakotensis 453. col. O.A. Stevens }\end{array}$ \\
\hline Zizanea aquatica & ME & & $11.9 \pm 1.7$ & $4.7 \pm 0.9$ & Ellipsoidal-oblong & NY: Orono, 1942, col. F.H. Steinmetz \\
\hline Zizanea aquatica & MN & & $12.7 \pm 2.9$ & $4.6 \pm 0.4$ & Ellipsoidal-oblong & OSC 97749 \\
\hline \multicolumn{7}{|l|}{ Chloridoideae } \\
\hline \multicolumn{7}{|l|}{ Chlorideae } \\
\hline \multicolumn{7}{|l|}{ Claviceps cinerea } \\
\hline Hilaria jamesii & NM & $\begin{array}{l}\text { Macro: } \\
\text { Micro: }\end{array}$ & $\begin{array}{l}20.2 \pm 4.6 \\
7.5 \pm 1.3\end{array}$ & $\begin{array}{l}5.0 \pm 0.7 \\
4.0 \pm 0.7\end{array}$ & Fusiform ellipsoidal & $\begin{array}{l}\text { NY: near Newkirk, 1941, col. D.A. Sav- } \\
\text { age, det. W.W. Ray, A\&M Herb. } 2872\end{array}$ \\
\hline Hilaria mutica & $\mathrm{AZ}$ & $\begin{array}{l}\text { Macro: } \\
\text { Micro: }\end{array}$ & $\begin{array}{c}22.2 \pm 3.7 \\
6.4 \pm 1.1\end{array}$ & $\begin{array}{l}5.1 \pm 0.5 \\
3.7 \pm 0.5\end{array}$ & Fusiform ellipsoidal & $\begin{array}{l}\text { NY: 1900, West American Fungi 97, } \\
\text { David Griffiths }\end{array}$ \\
\hline Hilaria mutica & $\mathrm{TX}$ & $\begin{array}{l}\text { Macro: } \\
\text { Micro: }\end{array}$ & $\begin{array}{c}25.6 \pm 2.9 \\
7.6 \pm 1.1\end{array}$ & $\begin{array}{l}4.8 \pm 0.5 \\
4.2 \pm 0.4\end{array}$ & Fusiform ellipsoidal & $\begin{array}{l}\text { FH: Sterling Co., 1944, col. A.F. Brat- } \\
\text { ton, Texas Ag. Exp. Stn. } 46102\end{array}$ \\
\hline \multicolumn{7}{|l|}{$\begin{array}{l}\text { Claviceps purpurea var. } \\
\text { spartinae }\end{array}$} \\
\hline Spartina alterniflora & MA & & $10.2 \pm 1.4$ & $3.9 \pm 0.5$ & Oblong-allantoid & $\begin{array}{l}\text { MSC: Woods Hole, 1881, W. Trelease } \\
\text { (labeled S. stricta) }\end{array}$ \\
\hline Spartina alterniflora & $\mathrm{SC}$ & & $12.7 \pm 2.4$ & $3.8 \pm 0.5$ & Oblong-allantoid & OSC 12011 (labeled Spartina sp.) \\
\hline Spartina pectinata & MT & & $17.1 \pm 3.0$ & $3.9 \pm 0.6$ & Oblong & OSC 69007 (labeled S. michauxiana) \\
\hline Spartina patens & NY & & $11.8 \pm 4.3$ & $4.3 \pm 0.5$ & Ellipsoidal-oblong & NY: Ex. NYS 27916 \\
\hline Panicoideae & & & & & & \\
\hline Andropogoneae & & & & & & \\
\hline Claviceps sp. & & & & & & \\
\hline Andropogon geradii & IA & Macro: & $11.8 \pm 1.0$ & $4.7 \pm 0.5$ & Ellipsoidal & ISC 319388 (labeled A. furcatus) \\
\hline Andropogon gerardii & $\mathrm{NM}$ & Micro: & $\begin{array}{c}3.9 \pm 0.6 \\
6.3 \pm 2.9 \\
\text { range: } 2-13\end{array}$ & $\begin{array}{c}3.0 \pm 0.2 \\
3.2 \pm 0.8 \\
\text { range: } 2-5\end{array}$ & $\begin{array}{l}\text { Spherical-ellipsoidal } \\
\text { Spherical-oblong }\end{array}$ & BPI 633703 (labeled A. furcatus) \\
\hline Andropogon hallii & IA & $\begin{array}{l}\text { Macro: } \\
\text { Micro: }\end{array}$ & $\begin{array}{l}11.4 \pm 1.4 \\
3.6 \pm 0.6\end{array}$ & $\begin{array}{l}4.8 \pm 0.7 \\
3.0 \pm 0.4\end{array}$ & $\begin{array}{l}\text { Ellipsoidal-oblong } \\
\text { Spherical-ellipsoidal }\end{array}$ & ISC 319389 \\
\hline Andropogon hallii & OK & Macro: & $\begin{array}{c}10.0 \pm 1.2 \\
36+0.6\end{array}$ & $4.5 \pm 0.7$ & Ellipsoidal-oblong & BPI 633706 \\
\hline Sorghastrum nutans & $\mathrm{TX}$ & Macro: & $8.3 \pm 0.9$ & $3.5 \pm 0.5$ & Ellipsoidal-oblong & BPI 634253 \\
\hline Claviceps africana & & & & & & \\
\hline Sorghum bicolor & $\mathrm{TX}$ & $\begin{array}{l}\text { Macro: } \\
\text { Micro: }\end{array}$ & $\begin{array}{l}14.0 \pm 1.5 \\
3.3 \pm 0.5\end{array}$ & $\begin{array}{l}4.9 \pm 0.5 \\
3.0 \pm 0.3\end{array}$ & $\begin{array}{l}\text { Ellipsoidal-oblong } \\
\text { Spherical }\end{array}$ & OSC 97751 \\
\hline Claviceps gigantae & & & & & & \\
\hline Zea maydis & (Mexico) & $\begin{array}{l}\text { Macro: } \\
\text { Micro: }\end{array}$ & $\begin{array}{c}24.7 \pm 5.1 \\
4.9 \pm 0.9\end{array}$ & $\begin{array}{l}4.3 \pm 0.7 \\
2.7 \pm 0.5\end{array}$ & $\begin{array}{l}\text { Fusiform } \\
\text { Ellipsiodal }\end{array}$ & $\begin{array}{l}\text { NY: near Mexico City, 1960, col. J.S } \\
\text { Niederhauser, det. C.T. Rogerson }\end{array}$ \\
\hline Claviceps tripsaci & & & & & & \\
\hline Tripsacum dactyloides & MD & Macro: & $26.6 \pm 5.6$ & $5.4 \pm 0.8$ & Fusiform & NY: near Chevy Chase, 1920, A. Chase \\
\hline & & Meso: & $11.4 \pm 1.6$ & $3.8 \pm 0.6$ & Ellipsoidal-oblong & \\
\hline & & Micro: & $4.3 \pm 0.8$ & $2.5 \pm 0.4$ & Spherical-ellipsoidal & \\
\hline Paniceae & & & & & & \\
\hline Claviceps digitareae & & & & & & \\
\hline Digitaria eriantha & $\mathrm{DC}$ & Macro: & $11.7 \pm 1.3$ & $4.6 \pm 0.7$ & Ellipsoidal-oblong & BPI 633148 \\
\hline & (S. Africa) & Micro: & $4.2 \pm 0.5$ & $3.2 \pm 0.3$ & Spherical-ellipsoidal & \\
\hline & & & & & & (Continued on next page) \\
\hline
\end{tabular}


Table 2. (Continued from previous page)

\begin{tabular}{|c|c|c|c|c|c|c|}
\hline Species & Location & $\begin{array}{c}\text { Conidial } \\
\text { type }\end{array}$ & $\begin{array}{c}\text { Conidial } \\
\text { length }(\mu \mathrm{m})\end{array}$ & $\begin{array}{c}\text { Conidial } \\
\text { width }(\mu \mathrm{m})\end{array}$ & Conidial shape & Source \\
\hline Digitaria valida & $\begin{array}{c}\text { DC } \\
\text { (S. Africa) }\end{array}$ & $\begin{array}{l}\text { Macro: } \\
\text { Micro: }\end{array}$ & $\begin{array}{l}11.7 \pm 2.1 \\
3.9 \pm 0.4\end{array}$ & $\begin{array}{l}4.8 \pm 1.0 \\
3.0 \pm 0.4\end{array}$ & $\begin{array}{l}\text { Ellipsoidal-oblong } \\
\text { Spherical-ellipsoidal }\end{array}$ & BPI 633035 \\
\hline \multicolumn{7}{|l|}{ Claviceps fusiformis } \\
\hline Pennisetum & (Rhodesia) & $\begin{array}{l}\text { Macro: } \\
\text { Meso: } \\
\text { Micro: }\end{array}$ & $\begin{array}{l}20.5 \pm 2.5 \\
5.5 \pm 0.7 \\
3.3 \pm 0.8\end{array}$ & $\begin{array}{l}4.9 \pm 0.6 \\
4.0 \pm 0.7 \\
2.5 \pm 0.5\end{array}$ & $\begin{array}{l}\text { Fusiform } \\
\text { Spherical-ellipsoidal } \\
\text { Spherical-ellipsoidal }\end{array}$ & IMI 110729 \\
\hline \multicolumn{7}{|l|}{ Claviceps maximensis } \\
\hline Panicum maximum & $\begin{array}{l}\text { (Puerto } \\
\text { Rico) }\end{array}$ & $\begin{array}{l}\text { Macro: } \\
\text { Micro: }\end{array}$ & $\begin{array}{c}15.8 \pm 4.7 \\
5.1 \pm 1.2\end{array}$ & $\begin{array}{l}5.9 \pm 0.9 \\
2.9 \pm 0.4\end{array}$ & $\begin{array}{l}\text { Ellipsoidal-oblong } \\
\text { Ellipsoidal }\end{array}$ & BPI 634487 \\
\hline $\begin{array}{l}\text { Claviceps paspali } \\
\text { Paspalum dilatatum }\end{array}$ & $\mathrm{AR}$ & & $12.2 \pm 1.7$ & $4.0 \pm 0.6$ & Ellipsoidal-oblong & $\begin{array}{l}\text { WIS: Little Rock, 1957, col. G. } \\
\text { Templeton, det. M.S. Pavgi }\end{array}$ \\
\hline $\begin{array}{l}\text { Paspalum plicatum } \\
\text { Claviceps } \mathrm{sp} \text {. }\end{array}$ & FL & & $12.3 \pm 1.4$ & $4.6 \pm 0.5$ & Ellipsoidal-oblong & UC 669553 \\
\hline Setaria macrostachya & NM & & $8.4 \pm 1.15$ & $3.9 \pm 0.6$ & Ellipsoidal & $\begin{array}{l}\text { NY: Carlsbad, 1902, Plants of the Gulf } \\
\text { States, col. S.M. Tracy (labeled } \\
\text { Chaetachloa composita) }\end{array}$ \\
\hline Setaria macrostachya & NM & & $7.5 \pm 1.3$ & $3.3 \pm 0.5$ & Ellipsoidal-oblong & $\begin{array}{l}\text { NY: Carlsbad, 1902, Plants of the Gulf } \\
\text { States, col. S.M. Tracy (labeled } S \text {. } \\
\text { composita) }\end{array}$ \\
\hline Setaria macrostachya & $\mathrm{TX}$ & $\begin{array}{l}\text { Macro: } \\
\text { Micro: }\end{array}$ & $\begin{array}{l}14.7 \pm 2.7 \\
4.9 \pm 1.2\end{array}$ & $\begin{array}{l}4.4 \pm 0.6 \\
3.1 \pm 0.3\end{array}$ & $\begin{array}{l}\text { Oblong-allantoid } \\
\text { Spherical-ellipsoidal }\end{array}$ & ICS 415193 \\
\hline Setaria valpiseta & $\mathrm{TX}$ & $\begin{array}{l}\text { Macro: } \\
\text { Micro: }\end{array}$ & $\begin{array}{l}18.0 \pm 2.1 \\
4.7 \pm 0.6\end{array}$ & $\begin{array}{l}6.1 \pm 0.9 \\
3.7 \pm 0.5\end{array}$ & $\begin{array}{l}\text { Oblong-allantoid } \\
\text { Spherical-ellipsoidal }\end{array}$ & FH 46065 (labeled S. macrostachya) \\
\hline \multicolumn{7}{|l|}{ Pooideae } \\
\hline $\begin{array}{l}\text { Claviceps purpurea } \\
\text { Agrostis exerata }\end{array}$ & OR & & $8.5 \pm 1.1$ & $4.1 \pm 0.5$ & Ellipsoidal & $\begin{array}{l}\text { NY: Warrenton, 1935, col. F.D. Bailey, } \\
\text { det. S.M. Zeller }\end{array}$ \\
\hline Agrostis stolonifera & IL & & $8.6 \pm 1.6$ & $4.1 \pm 0.5$ & Ellipsoidal & ILLS 33788 (labeled A. alba) \\
\hline Agrostis. stolonifera & OR & & $7.5 \pm 1.1$ & $3.6 \pm 0.4$ & Ellipsoidal & OSC 10722 (labeled A. alba) \\
\hline Alopecuris geniculatus & ND & & $8.3 \pm 1.1$ & $4.0 \pm 0.5$ & Ellipsoidal & RM 18886 \\
\hline Alopecuris pratensis & MI & & $9.3 \pm 1.3$ & $4.2 \pm 0.6$ & Ellipsoidal-oblong & MSC: Walton Cr, Traverse Co., 1889 \\
\hline Alopecuris pratensis & OR & & $9.1 \pm 1.3$ & $3.8 \pm 0.6$ & Ellipsoidal-oblong & NY: Astoria, 1936, R.S. Sprague \\
\hline Ammophila breviligulata & NY & & $6.5 \pm 1.1$ & $3.6 \pm 0.5$ & Ellipsoidal & NY: Ex NYS 27934 \\
\hline Ammophila breviligulata & NY & & $9.9 \pm 2.8$ & $4.1 \pm 0.7$ & Ellipsoidal-oblong & BPI 633717 \\
\hline Anthoxanthum odoratum & NY & & $7.0 \pm 1.6$ & $3.1 \pm 0.4$ & Ellipsoidal-oblong & $\begin{array}{l}\text { NY: Ulster Co., 1988, Fungi of New } \\
\text { York 571, col. R. Lowen }\end{array}$ \\
\hline Avena sativa & NY & & $7.2 \pm 1.0$ & $3.4 \pm 0.4$ & Ellipsoidal & CUP 37862 \\
\hline Calamagrostis canadensis & $\mathrm{AK}$ & & $11.9 \pm 1.8$ & $4.3 \pm 0.6$ & Ellipsoidal-oblong & $\begin{array}{l}\text { WIS: Matanuska, 1956, col. J.G. Dick- } \\
\text { son }\end{array}$ \\
\hline Calamagrostis canadensis & $\mathrm{AK}$ & & $10.1 \pm 1.6$ & $4.3 \pm 0.5$ & Ellipsoidal-oblong & WSP 51546 \\
\hline Calamagrostis canadensis & ME & & $10.3 \pm 1.6$ & $3.8 \pm 0.5$ & Ellipsoidal-oblong & CUP 32951 \\
\hline Calamagrostis canadensis & $\mathrm{MN}$ & & $11.6 \pm 2.3$ & $4.7 \pm 0.8$ & Ellipsoidal-oblong & $\begin{array}{l}\text { MSC: Minneapolis, 1883, Herb. W.J. } \\
\text { Beal labeled (Deyeuxia canadensis) }\end{array}$ \\
\hline Calamagrostis canadensis & MN & & $8.5 \pm 1.0$ & $4.0 \pm 0.5$ & Ellipsoidal & WIS: Waterville, 1905, J.H. Kearnen \\
\hline Calamagrostis canadensis & NY & & $10.4 \pm 1.8$ & $4.4 \pm 0.5$ & Ellipsoidal-oblong & CUP 23904 \\
\hline Calamagrostis canadensis & WA & & $11.2 \pm 1.6$ & $4.0 \pm 0.5$ & Ellipsoidal-oblong & $\begin{array}{l}\text { UC } 620739 \text { (labeled Deschampsia atro- } \\
\text { purpurea) }\end{array}$ \\
\hline Calamagrostis stricta & ND & & $9.3 \pm 2.0$ & $4.0 \pm 0.7$ & Ellipsoidal-oblong & $\begin{array}{l}\text { NY: Kulm, 1908, North Dakota Fungi, } \\
\text { col J. F. Brenckle (labeled C. neglecta) }\end{array}$ \\
\hline $\begin{array}{l}\text { Calamagrostis stricta } \mathrm{ssp} . \\
\text { inexpansa }\end{array}$ & MN & & $10.8 \pm 2.1$ & $4.7 \pm 0.4$ & Ellipsoidal-oblong & WSP 46641 (labeled C. inexpansa) \\
\hline Cinna latifolia & $\mathrm{AK}$ & & $11.7 \pm 0.9$ & $4.2 \pm 0.6$ & Ellipsoidal-oblong & WSP 51116 \\
\hline Cinna latifolia & Canada & & $10.0 \pm 1.8$ & $3.9 \pm 0.8$ & Ellipsoidal-oblong & CUP 19159 \\
\hline Holcus lanatus & OR & & $8.5 \pm 1.1$ & $4.1 \pm 0.4$ & Ellipsoidal & OSC 1790 \\
\hline Holcus lanatus & WA & & $8.9 \pm 1.5$ & $3.8 \pm 0.4$ & Ellipsoidal-oblong & CUP 33142 \\
\hline Koeleria macrantha & $\mathrm{AZ}$ & & $7.2 \pm 1.0$ & $3.2 \pm 0.4$ & Ellipsoidal & $\begin{array}{l}\text { WIS: Bradshaw Mts., 1892, J.W. Tour- } \\
\text { mey (labeled K. cristata) }\end{array}$ \\
\hline Phalaris arundinacea & IA & & $9.0 \pm 1.8$ & $4.6 \pm 0.8$ & Ellipsoidal & ISC 318625 (labeled Koeleria cristata) \\
\hline Phalaris arundinacea & MA & & $8.8 \pm 1.4$ & $3.4 \pm 0.5$ & Ellipsoidal-oblong & $\begin{array}{l}\text { NY: Hadley, 1978, col. H.E. Ahlees, det } \\
\text { M.E.B.B. }\end{array}$ \\
\hline Phalaris arundinacea & MA & & $9.1 \pm 0.8$ & $4.1 \pm 0.5$ & Ellipsoidal-oblong & $\begin{array}{l}\text { FH: Newton, 1935, P.L. Rusdon (labeled } \\
\text { Calamagrostis cinnoides) }\end{array}$ \\
\hline Phalaris arundinacea & MI & & $8.6 \pm 1.2$ & $3.8 \pm 0.4$ & Ellipsoidal-oblong & MICH: Chelsea, 1927, D.M. Benedict \\
\hline Phalaris arundinacea & VA & & $8.6 \pm 1.1$ & $3.8 \pm 0.4$ & Ellipsoidal-oblong & $\begin{array}{l}\text { FH: Paris, 1923, col. C. drechsler, det. } \\
\text { V.K. Charles }\end{array}$ \\
\hline Phalaris arundinacea & NY & & $9.3 \pm 1.5$ & $4.1 \pm 0.4$ & Ellipsoidal-oblong & CUP 10007 \\
\hline Phalaris arundinacea & VA & & $8.8 \pm 1.8$ & $3.4 \pm 0.6$ & Ellipsoidal-oblong & CUP 13671 \\
\hline & IL & & $6.9 \pm 0.9$ & $3.6 \pm 0.5$ & Ellipsoidal & F 1166637 \\
\hline \multirow[t]{2}{*}{ Phleum pratense } & OR & & $7.9 \pm 0.8$ & $3.6 \pm 0.4$ & Ellipsoidal & OSU 10014 \\
\hline & & & & & & (Continued on next page) \\
\hline
\end{tabular}


Table 2. (Continued from previous page)

\begin{tabular}{|c|c|c|c|c|c|c|}
\hline Species & Location & $\begin{array}{c}\text { Conidial } \\
\text { type }\end{array}$ & $\begin{array}{c}\text { Conidial } \\
\text { length }(\mu \mathrm{m})\end{array}$ & $\begin{array}{c}\text { Conidial } \\
\text { width }(\mu \mathrm{m})\end{array}$ & Conidial shape & Source \\
\hline Phleum pratense & OR & & $9.1 \pm 1.8$ & $4.1 \pm 0.6$ & Ellipsoidal-oblong & OSC 1731 \\
\hline Phleum pratense & WI & & $9.4 \pm 1.6$ & $4.3 \pm 0.5$ & Ellipsoidal & CUP 4750 \\
\hline \multicolumn{7}{|l|}{ Bromeae } \\
\hline \multicolumn{7}{|l|}{ Claviceps purpurea } \\
\hline Bromus carinatus & $\mathrm{CA}$ & & $6.4 \pm 0.8$ & $3.9 \pm 0.4$ & Ellipsoidal & $\begin{array}{l}\text { F: Oxford Tract, Berkeley, 1949, Fungi } \\
\text { of CA, col. A. R. Kruckeberg (labeled } \\
\text { B. marginatus) }\end{array}$ \\
\hline Bromus carinatus & ID & & $6.4 \pm 0.8$ & $3.6 \pm 0.4$ & Ellipsoidal & WSP 16633 \\
\hline Bromus carinatus & WA & & $6.2 \pm 1.4$ & $3.5 \pm 0.4$ & Ellipsoidal & WSP 21509 \\
\hline Bromus carinatus & WA & & $5.6 \pm 0.7$ & $2.8 \pm 0.3$ & Ellipsoidal & WSP 13385 \\
\hline Bromus commutatus & IL & & $6.7 \pm 1.0$ & $3.6 \pm 0.4$ & Ellipsoidal & ILLS 27874 (labeled B. secalinus) \\
\hline Bromus inermis & $\mathrm{IL}$ & & $6.9 \pm 1.0$ & $3.6 \pm 0.6$ & Ellipsoidal & ILLS 29117 \\
\hline Bromus inermis & WA & & $5.8 \pm 0.7$ & $3.2 \pm 0.4$ & Ellipsoidal & WSP 19934 \\
\hline \multicolumn{7}{|l|}{ Meliceae } \\
\hline \multicolumn{7}{|l|}{ Claviceps purpurea } \\
\hline Glyceria borealis & MI & & $10.8 \pm 1.9$ & $4.7 \pm 0.7$ & Ellipsoidal-oblong & $\begin{array}{l}\text { MICH: McCargo Cove, 1930, col. C.A. } \\
\text { Brown, det. E.B. Mains }\end{array}$ \\
\hline Glyceria septentrionalis & IA & & $10.5 \pm 0.9$ & $3.7 \pm 0.4$ & Ellipsoidal-oblong & ISC 318655 \\
\hline Glyceria $\times$ Laxa & Canada & & $10.1 \pm 1.7$ & $3.9 \pm 0.6$ & Ellipsoidal-oblong & FH 18139 (labeled Glyceria canadensis) \\
\hline Glyceria borealis & NY & & $10.7 \pm 1.8$ & $4.3 \pm 0.6$ & Ellipsoidal-oblong & CUP 29423 \\
\hline Glyceria fluitans & Germany & & $8.7 \pm 1.1$ & $3.7 \pm 0.4$ & Ellipsoidal-oblong & OSC 37892 \\
\hline Glyceria fluitans & Canada & & $11.3 \pm 1.2$ & $4.2 \pm 0.7$ & Ellipsoidal-oblong & CUP 4516 \\
\hline \multicolumn{7}{|l|}{ Poeae } \\
\hline \multicolumn{7}{|l|}{ Claviceps purpurea } \\
\hline Dactylis glomerata & IA & & $7.2 \pm 1.0$ & $3.6 \pm 0.5$ & Ellipsoidal & ICS 318613 \\
\hline Dactylis glomerata & IL & & $5.9 \pm 0.7$ & $3.4 \pm 0.5$ & Ellipsoidal & ILLS 25945 \\
\hline Dactylis glomerata & MI & & $5.9 \pm 0.8$ & $3.1 \pm 0.4$ & Ellipsoidal & MSC: East lansing, 1930, J. P. Kienholz \\
\hline Dactylis glomerata & NY & & $6.5 \pm 1.2$ & $3.0 \pm 0.4$ & Ellipsoidal & CUP 35966 \\
\hline Dactylis glomerata & WI & & $7.4 \pm 1.5$ & $3.8 \pm 0.6$ & Ellipsoidal & WIS: Madison, 1919, J.J. Davis \\
\hline Lolium multiflorum & OR & & $7.5 \pm 0.9$ & $3.8 \pm 0.5$ & Ellipsoidal & OSC 1743 (labeled Lolium italicum) \\
\hline Lolium multiflorum & OR & & $5.9 \pm 0.7$ & $3.3 \pm 0.3$ & Ellipsoidal & OSC 10744 \\
\hline Lolium multiflorum & OR & & $6.0 \pm 0.5$ & $3.3 \pm 0.4$ & Ellipsoidal & OSC 10698 \\
\hline Lolium perenne & MA & & $6.9 \pm 1.2$ & $3.4 \pm 0.5$ & Ellipsoidal & $\begin{array}{l}\text { NY: Amherst, 1967, col. H.E. Ahles, } \\
\text { det. M.E.B.B. }\end{array}$ \\
\hline Lolium perenne & OR & & $6.8 \pm 0.9$ & $3.7 \pm 0.3$ & Ellipsoidal & OSC 39275 \\
\hline Lolium perenne & WY & & $7.1 \pm 0.9$ & $3.6 \pm 0.5$ & Ellipsoidal & NY: Corvallis, 1915, H.C. Gilbert \\
\hline Lolium pratense & MA & & $6.9 \pm 1.2$ & $3.3 \pm 0.6$ & Ellipsoidal & $\begin{array}{l}\text { NY: Amherst, 1967, Col. H.E. Ahles, det. } \\
\text { M.E.B.B. (labeled Festuca elatior) }\end{array}$ \\
\hline Lolium arundinaceum & NY & & $6.0 \pm 0.7$ & $3.0 \pm 0.5$ & & CUP 9955 (labeled Festuca elatior) \\
\hline Poa fendleriana & UT & & $7.6 \pm 1.3$ & $3.9 \pm 0.4$ & Ellipsoidal & $\begin{array}{l}\text { NY: Fungi Columbiani 2317, E. Bar- } \\
\text { tholomew (labeled Poa longiligula) }\end{array}$ \\
\hline Poa secunda ssp. juncifolia & $\mathrm{CA}$ & & $5.7 \pm 0.9$ & $3.1 \pm 0.4$ & Ellipsoidal & WSP 25499 (labeled Poa nevadensis) \\
\hline Poa pratensis & OR & & $8.5 \pm 0.8$ & $3.3 \pm 0.3$ & Ellipsoidal-oblong & OSC 97750 \\
\hline Poa secunda & ID & & $5.8 \pm 0.6$ & $3.3 \pm 0.4$ & Ellipsoidal & WSP 42803 (labeled Poa ampla) \\
\hline Poa secunda & UT & & $6.9 \pm 1.0$ & $3.6 \pm 0.4$ & Ellipsoidal & WSP 13383 (labeled Poa scabella) \\
\hline \multicolumn{7}{|l|}{ Triticeae } \\
\hline \multicolumn{7}{|l|}{ Claviceps purpurea } \\
\hline Agropyron cristatum & WA & & $6.8 \pm 1.1$ & $3.7 \pm 0.5$ & Ellipsoidal & $\begin{array}{l}\text { NY: Mycobiota of North America 304, } \\
\text { 1948, col. \& det. J. Meiners }\end{array}$ \\
\hline Elymus canadensis & IL & & $6.0 \pm 0.6$ & $2.8 \pm 0.3$ & Ellipsoidal & ILLS 30054 \\
\hline Elymus canadensis & KS & & $6.1 \pm 0.9$ & $3.2 \pm 0.8$ & Ellipsoidal & $\begin{array}{l}\text { NY: Rooks, Co. 1902, Ellis and } \\
\text { Everhart's Fungi Columbiani } 1614\end{array}$ \\
\hline Elymus canadensis & OR & & $6.2 \pm 0.8$ & $3.6 \pm 0.4$ & Ellipsoidal & OSC 1804 \\
\hline Elymus canadensis & SD & & $6.2 \pm 1.1$ & $3.8 \pm 0.5$ & Ellipsoidal & NY: Farmer, 1927, G.F. Weber \\
\hline Elymus canadensis & WI & & $6.1 \pm 0.7$ & $2.9 \pm 0.5$ & Ellipsoidal & $\begin{array}{l}\text { WIS: Trempealeau, 1914, R.H. Dennis- } \\
\text { ton }\end{array}$ \\
\hline Elymus canadensis & WI & & $6.0 \pm 0.8$ & $3.4 \pm 0.4$ & Ellipsoidal & $\begin{array}{l}\text { WIS: Madison, 1893, Fungi of the T.A. } \\
\text { Williams Collection }\end{array}$ \\
\hline Elymus caninus & IA & & $5.6 \pm 0.7$ & $3.0 \pm 0.3$ & Ellipsoidal & $\begin{array}{l}\text { ICS } 386395 \text { (labeled Agropyron can- } \\
\text { inum) }\end{array}$ \\
\hline Elymus caninus & MI & & $7.9 \pm 1.4$ & $3.4 \pm 0.6$ & Ellipsoidal-oblong & $\begin{array}{l}\text { MSC: Ag. Coll., } 1889 \text { (labeled Agropy- } \\
\text { ron caninum) }\end{array}$ \\
\hline Elymus glaucus & OR & & $6.2 \pm 0.7$ & $3.4 \pm 0.5$ & Ellipsoidal & OSC 13669 \\
\hline Elymus glaucus & OR & & $5.6 \pm 0.6$ & $3.3 \pm 0.4$ & Ellipsoidal & WSP 21022 \\
\hline Elymus glaucus & WY & & $6.2 \pm 0.7$ & $3.5 \pm 0.4$ & Ellipsoidal & RM 26034 \\
\hline Elymus lanceolatus & WY & & $5.9 \pm 0.7$ & $3.8 \pm 0.4$ & Ellipsoidal & $\begin{array}{l}\text { NY: Mycoflora Saximontanensis Exsic- } \\
\text { cata 122, 1935, W.G. Solheim (labeled } \\
\text { as Agropyron dasystachyum) }\end{array}$ \\
\hline $\begin{array}{l}\text { Elymus } \times \text { Agrohordeum } \\
\text { macouni }\end{array}$ & $\mathrm{NE}$ & & $6.8 \pm 0.9$ & $3.9 \pm 0.6$ & Ellipsoidal & NEB 158700 (labeled Elymus macouni) \\
\hline \multirow[t]{2}{*}{ Elymus macounii } & WA & & $5.9 \pm 0.6$ & $3.5 \pm 0.4$ & Ellipsoidal & WSP 13384 \\
\hline & & & & & & (Continued on next page) \\
\hline
\end{tabular}


Table 2. (Continued from previous page)

\begin{tabular}{|c|c|c|c|c|c|c|}
\hline Species & Location & $\begin{array}{l}\text { Conidial } \\
\text { type }\end{array}$ & $\begin{array}{l}\text { Conidial } \\
\text { length }(\mu \mathrm{m})\end{array}$ & $\begin{array}{c}\text { Conidial } \\
\text { width }(\mu \mathrm{m})\end{array}$ & Conidial shape & Source \\
\hline Elymus repens & NE & & $5.8 \pm 1.0$ & $3.4 \pm 0.4$ & Ellipsoidal & $\begin{array}{l}\text { NEB } 158701 \text { (labeled Agropyron pseu- } \\
\text { dorepens) }\end{array}$ \\
\hline Elymus virginicus & IA & & $5.8 \pm 0.8$ & $2.5 \pm 0.4$ & Ellipsoidal-oblong & UC: Awana, 1925, B. Shimek \\
\hline Elymus virginicus & IL & & $6.1 \pm 1.1$ & $2.8 \pm 0.5$ & Ellipsoidal & ILLS 22045 \\
\hline Elymus virginicus & $\mathrm{NE}$ & & $6.0 \pm 1.2$ & $2.8 \pm 0.3$ & Ellipsoidal & $\begin{array}{l}\text { NY: Red Cloud, 1906, Fungi Columbi- } \\
\text { ani 2216, E. Bartholomew }\end{array}$ \\
\hline Elytrigia intermedia & KS & & $7.2 \pm 1.3$ & $3.3 \pm 0.7$ & Ellipsoidal & $\begin{array}{l}\text { NY: Rockport, 1892, Kansas Fungi, E. } \\
\text { Bartholomew (labeled Agropyrum } \\
\text { glaucum) }\end{array}$ \\
\hline Elytrigia repens & IL & & $6.5 \pm 1.0$ & $3.4 \pm 0.4$ & Ellipsoidal & ILLS 28732 (labeled Agropyron repens) \\
\hline Elytrigia repens & MA & & $6.2 \pm 1.2$ & $3.6 \pm 0.5$ & Ellipsoidal & $\begin{array}{l}\text { NY: Amherst, 1925, Massachusetts } \\
\text { Fungi 3012, T.T. Ayres \& W.L. Doran } \\
\text { (labeled Agropyron repens) }\end{array}$ \\
\hline Elytrigia repens & OR & & $5.9 \pm 0.8$ & $3.2 \pm 0.4$ & Ellipsoidal & $\begin{array}{l}\text { NY: Hood River, 1936, J.R. Kienholz } \\
\text { (labeled Agropyron repens) }\end{array}$ \\
\hline Elytrigia repens & NY & & $6.9 \pm 0.9$ & $3.9 \pm 0.5$ & Ellipsoidal & $\begin{array}{l}\text { NY: Ex NYS } 1041 \text { (labeled Agropyron } \\
\text { repens) }\end{array}$ \\
\hline Elytrigia repens & PA & & $6.1 \pm 1.4$ & $3.1 \pm 0.2$ & Ellipsoidal & WSP1094 (labeled Agropyron repens) \\
\hline Elytrigia repens & VT & & $6.4 \pm 1.0$ & $3.9 \pm 0.5$ & Ellipsoidal & FLAS F46374 (labeled Agropyron repens) \\
\hline Elytrigia repens & WI & & $6.3 \pm 0.9$ & $3.3 \pm 0.5$ & Ellipsoidal & $\begin{array}{l}\text { WIS: Butternut, 1911, J.J. Davis (la- } \\
\text { beled Agropyron repens) }\end{array}$ \\
\hline Elytrigia smithii & MA & & $6.4 \pm 0.9$ & $3.7 \pm 0.4$ & Ellipsoidal & $\begin{array}{l}\text { NY: Amherst, 1966, H.E. Ahles (labeled } \\
\text { Agropyron smithii) }\end{array}$ \\
\hline Hordeum vulgare & WA & & $6.5 \pm 1.3$ & $4.3 \pm 0.6$ & Ellipsoidal & WSP 1114 \\
\hline Leymus cinereus & WA & & $6.0 \pm 0.8$ & $3.3 \pm 0.4$ & Ellipsoidal & WSP 16443 \\
\hline Leymus cinereus & $\mathrm{CO}$ & & $6.7 \pm 0.9$ & $3.4 \pm 0.4$ & Ellipsoidal & OSC 6339 (labeled Elymus sp.) \\
\hline Leymus cinereus & OR & & $5.5 \pm 0.7$ & $3.6 \pm 0.5$ & Ellipsoidal & OSC 10694 (labeled Elymus condensatus) \\
\hline Leymus cinereus & UT & & $5.6 \pm 0.7$ & $3.3 \pm 0.5$ & Ellipsoidal & $\begin{array}{l}\text { NY: Weber Co., 1985, C.T. Rogerson } \\
\text { (labeled Elymus cinereus) }\end{array}$ \\
\hline Leymus cinereus & ID & & $5.4 \pm 0.5$ & $3.2 \pm 0.3$ & Ellipsoidal & WSP 46010 (labeled Elymus cinereus) \\
\hline Leymus cinereus & WY & & $6.3 \pm 0.6$ & $3.5 \pm 0.5$ & Ellipsoidal & UC 201420 (labeled Elymus cinereus) \\
\hline Leymus condensatus & $\mathrm{CO}$ & & $6.6 \pm 0.8$ & $3.9 \pm 0.3$ & Ellipsoidal & RM 14584 (labeled Elymus condensatus) \\
\hline Leymus condensatus & OR & & $5.7 \pm 0.7$ & $3.4 \pm 0.5$ & Ellipsoidal & $\begin{array}{l}\text { NY: Echo, 1937, R. Sprague (labeled } \\
\text { Elymus condensatus) }\end{array}$ \\
\hline Leymus condensatus & WY & & $5.6 \pm 0.7$ & $3.3 \pm 0.4$ & Ellipsoidal & $\begin{array}{l}\text { NY: Fungi Columbiani 3704, E. Bar- } \\
\text { tholomew (labeled Elymus conden- } \\
\text { satus) }\end{array}$ \\
\hline Leymus condensatus & WY & & $6.3 \pm 0.9$ & $3.6 \pm 0.4$ & Ellipsoidal & RM 140953 (labeled Elymus condensatus) \\
\hline Leymus triticoides & OR & & $5.9 \pm 0.9$ & $3.5 \pm 0.4$ & Ellipsoidal & WSP 46004 (labeled Eylmus triticoides) \\
\hline Leymus triticoides & OR & & $5.5 \pm 0.7$ & $3.4 \pm 0.4$ & Ellipsoidal & OSC 10689 (labeled Elymus triticoides) \\
\hline Pascopyrum smithii & MT & & $7.2 \pm 1.2$ & $3.8 \pm 0.4$ & Ellipsoidal & $\begin{array}{l}\text { OSC: Fungi Columbiani } 4311, \text { E. Bar- } \\
\text { tholomew (labeled Agropyron occiden- } \\
\text { tale) }\end{array}$ \\
\hline Pascopyrum smithii & $\mathrm{NE}$ & & $6.4 \pm 0.9$ & $3.5 \pm 0.5$ & Ellipsoidal & $\begin{array}{l}\text { NEB } 1508705 \text { (labeled Agropyron occi- } \\
\text { dentale) }\end{array}$ \\
\hline Pascopyrum smithii & $\mathrm{NE}$ & & $6.2 \pm 0.8$ & $3.7 \pm 0.4$ & Ellipsoidal & $\begin{array}{l}\text { NEB } 158693 \text { (labeled Agropyron } \\
\text { smithii) }\end{array}$ \\
\hline Pascopyrum smithii & IA & & $6.0 \pm 1.2$ & $2.9 \pm 0.5$ & Ellipsoidal & $\begin{array}{l}\text { NY: W. Okaboju, 1932, G.W. Martin } \\
\text { (labeled Agropyron smithii) }\end{array}$ \\
\hline Pascopyrum smithii & $\mathrm{KS}$ & & $5.2 \pm 0.7$ & $2.9 \pm 0.5$ & Ellipsoidal & $\begin{array}{l}\text { NY: Ellis and Everhart's Fungi Colum- } \\
\text { biani 1816, E. Bartholomew (labeled } \\
\text { Agropyron occidentale) }\end{array}$ \\
\hline Secale cereale & ND & & $5.8 \pm 1.1$ & $3.2 \pm 0.4$ & Ellipsoidal & NY: Fungi Dakotenses 328, J.F. Brenckle \\
\hline Secale cereale & NY & & $6.5 \pm 0.9$ & $3.1 \pm 0.0$ & Ellipsoidal & CUP 2891 \\
\hline Triticum aestivum & NY & & $5.4 \pm 0.5$ & $3.3 \pm 0.3$ & Ellipsoidal & CUP 19373 \\
\hline \multicolumn{7}{|l|}{ Cyperaceae } \\
\hline \multicolumn{7}{|l|}{ Claviceps nigricans } \\
\hline Eleocharis macrostachya & $\mathrm{CA}$ & & $9.5 \pm 2.0$ & $3.8 \pm 0.5$ & Ellipsoidal-oblong & RM 38811 \\
\hline Eleocharis macrostachya & $\mathrm{CA}$ & & $10.4 \pm 0.9$ & $3.6 \pm 0.4$ & Ellipsoidal-oblong & CUP 47305 \\
\hline Eleocharis palustris & $\mathrm{CA}$ & & $11.2 \pm 2.1$ & $3.6 \pm 0.6$ & Ellipsoidal-oblong & $\begin{array}{l}\text { NY: CA Fungi 764, 1949, M.A. Nobs \& } \\
\text { S.G. Smith }\end{array}$ \\
\hline $\begin{array}{l}\text { Eleocharis rostellata } \\
\text { Claviceps grohii }\end{array}$ & $\mathrm{CA}$ & & $11.1 \pm 1.5$ & $3.6 \pm 0.4$ & Ellipsoidal-oblong & CUP 36613 \\
\hline Carex sp. & OR & & $16.4 \pm 1.9$ & $5.2 \pm 0.6$ & Ellipsoidal-oblong & OSC 1852 \\
\hline Carex brunnesens & Canada & & $11.2 \pm 1.3$ & $3.8 \pm 0.3$ & Ellipsoidal-oblong & DOAM 13621 \\
\hline $\begin{array}{l}\text { Carex canescens spp. arc- } \\
\text { taeformis }\end{array}$ & Canada & & $13.2 \pm 2.1$ & $4.5 \pm 0.5$ & Ellipsoidal-oblong & DOAM 105889 \\
\hline \multicolumn{7}{|l|}{ Juncaceae } \\
\hline \multicolumn{7}{|l|}{ Claviceps sp. } \\
\hline Juncus nevadensis & CA & & $12.1 \pm 1.5$ & $4.4 \pm 0.6$ & Ellipsoidal-oblong & $\begin{array}{l}\text { WIS: Modoc Co., 1958, col. L.C. } \\
\text { Anderson, det. H.C. Green }\end{array}$ \\
\hline Juncus ensifolius & Canada & & $13.8 \pm 2.1$ & $4.4 \pm 0.4$ & Ellipsoidal-oblong & NY: DOAM 214361 \\
\hline Juncus articulatus & Canada & & $12.7 \pm 1.7$ & $4.1 \pm 0.4$ & Ellipsoidal-oblong & FH: DOAM 184499 \\
\hline
\end{tabular}


gerardii Vitman, A. halli Hack., Bothriochloa ischaemum (L.) Keng., and Schizachyrium scoparium (Michx.) Nash. Yellow stipes and capitula observed in a collection from $B$. ischaemum from TX (BPI 633256) were consistent with descriptions of $C$. pusilla. However, in examination of specimens of Andropogon spp. from IA (Table 2), conidia were ellipsoidal, and the triangular conidia unique to $C$. pusilla were not seen.

C. ranunculoides was reported from AZ, NM, and TX on Setaria macrostachya Kunth, although the occurrence of $C$. ranunculoides in the United States is questioned (Table 1). Sclerotia of Claviceps on Setaria from TX and NM were cream to buff-brown to gray, with the upper half generally darker than the lower half, elongate, curved, slightly flattened, with a single longitudinal furrow on each flattened side and a relatively smooth texture. The specimens, labeled as $C$. ranunculoides, differed from blue-black sclerotia of the type description of C. ranunculoides (112), but they were somewhat similar to descriptions of C. sulcata Langdon from Brachiaria spp. (102). C. sulcata is not known to occur in the United States.

Claviceps tripsaci was reported from GA, DC, MD, MS, NC, OK, and VA. $C$. tripsaci is known only from Tripsacum dactyloides (L.) L. Three conidial types were recovered from sclerotia: fusiform macroconidia, $27 \times 5 \mu \mathrm{m}$, ellipsoidal mesoconidia, $11 \times 4 \mu \mathrm{m}$, and sphericalellipsoidal microconidia, $4 \times 3 \mu \mathrm{m}$.

Claviceps yanagawensis is known from $\mathrm{Fl}$ and VA on Zoysia japonica Steudal. Conidia were not observed in sufficient numbers for size determination.

C. zizaniae was reported from IA, ME, MD, MN, MO, NE, ND, and WI. Zizania aquatica L. and Z. palustris L. var. palustris are the only known hosts. Conidia of C. zizaniae from Zizania were ellipsoidal to oblong, 11-12 $\times 4-5 \mu \mathrm{m}$, with ends more tapered than rounded (Table 2).

\section{DISCUSSION}

The occurrence and distribution of Claviceps spp. in the United States reflects adaptation of Claviceps to a diversity of habitats, ranging from marine to arid and subtropical to northern temperate. Most Claviceps spp. have a narrow host range, including one to several closely related genera. The exception is $C$. purpurea var. purpurea. The vast majority of hosts for $C$. purpurea var. purpurea are in the Pooideae, with some hosts reported in the Arundinoideae. Genera in the Panicoideae are not considered hosts for $C$. purpurea var. purpurea (96), and our observations support this concept. The distribution of $C$. purpurea var. purpurea in the United States closely follows the distribution of the Pooideae, which include primarily cool season, temperate grasses. C. purpurea var. purpurea requires a period of several weeks of cold, near-freezing temperatures to induce fructification (111) and is therefore adapted to temperate climatic conditions. In addition, sclerotia of $C$. purpurea var. purpurea were reported susceptible to microbial decay under warmer conditions in the southeastern United States (28). However, C. purpurea var. purpurea may survive in the southeastern United States in the conidial state.

Because C. purpurea var. purpurea has such a wide host range and genetic diversity, it is often assumed to be the causal agent when floral sclerotia are found on a grass host. Confirmation of $C$. purpurea var. purpurea, especially on hosts outside the Pooideae, should be based on examination of sclerotia, ascostromata, and conidia, or through the determination and analysis of ITS and/or other sequences. Loveless $(100,101)$ found evidence for restricted host range among Claviceps spp. based on conidial morphology. We found conidial size to be a relatively stable attribute among species of Claviceps. However, consistently larger conidia developed on hosts in the tribes Aveneae and Meliceae than on hosts in other tribes in the Pooideae. Loveless and Peach (103) found conidial size to be under host genotype control, which suggests adaptation of strains of $C$. purpurea var. purpurea to specific hosts. However, Pazoutova et al. (119) found strains of $C$. purpurea var. purpurea adapted to various habitats, with larger conidia associated with hosts in wet habitats. Variation in habitat may account for the variability in conidial size observed in C. purpurea var. purpurea found on grasses in the Aveneae and Meliceae. Many of the grasses in the Aveneae (Calamagrostis) and Meliceae (Glyceria) on which large conidia of $C$. purpurea var. purpurea were found occur in wetland habitats. Some grass species (Calamagrostis canadensis, Cinna latifolia), associated with a large variation in size of conidia of $C$. purpurea var. purpurea, grow in both wetland and upland sites.

In our examination of Claviceps from the Panicoideae, tribe Andropogoneae, collections from Andropogon and Sorghastrum differed from $C$. purpurea var. purpurea by the presence of both macroconidia and microconidia, which clearly separated them from $C$. purpurea var. purpurea. In addition, sclerotia from the Andropogoneae were light in color compared with the dark colored sclerotia of $C$. purpurea var. purpurea. We agree with Langdon (96) that hosts in the panicoid grasses are likely not susceptible to $C$. purpurea var. purpurea. From an ecological perspective, this makes sense since the cold requirement for germination of sclerotia of C. purpurea var. purpurea would not be met by the subtropical habitat of many of the panicoid grasses.

A Claviceps sp. on Setaria from TX formed macroconidia and microconidia.
Collections from NM were similar except that clearly differentiated macroconidia were not observed. Since sclerotia were similar and unique in coloration and morphology, it is likely that macroconidia production was not as great in collections from NM. The Claviceps sp. on Setaria remains unidentified.

C. paspali was originally described from Paspalum in the United States by Stevens and Hall (153). It is widespread in the southern states, and it is an important pathogen of Paspalum spp. grown there for seed.

The earliest record found for C. pusilla was by Pammel and Weems in 1900 (116). Tiffany (157) identified C. pusilla on Andropogon spp. based on examination of ascomata from germinated sclerotia. Examination of a collection from TX (BPI) revealed yellow capitula characteristic of C. pusilla. However, in collections of Tiffany, labeled as $C$. pusilla, triangular conidia characteristic of this species were not present. Additional collections need to be examined to confirm the presence of $C$. pusilla in the United States. C. pusilla has a wide geographical distribution, is known from Andropogon spp., and likely occurs on Andropogon spp. in the United States. However, we consider reports of $C$. purpurea var. purpurea on Andropogon spp. to be incorrect.

C. ranunculoides was first described by Möller (112) on Setaria in Brazil. In the United States, it was reported on S. macrostachya from AZ, NM, and TX, but we consider these reports erroneous. The sclerotia from U.S. collections were gray in color and clearly differed from the dark, purple-black color described for sclerotia of $C$. ranunculoides. Therefore, the occurrence of $C$. ranunculoides in the United States is questionable. Setaria is a member of the Panicoideae, which includes hosts susceptible to several subtropical to tropical species of Claviceps. However, Setaria spp. are not considered hosts of $C$. purpurea var. purpurea.

C. tripsaci was first described from NC by Stevens and Hall (153) in 1910. Few reports of $C$. tripsaci occur, few collections are available, and little is known about this species beyond the original technical description of Stevens and Hall (153).

In the case of Claviceps on Zizania, studies of Fyles (46), Pantidou (117), and Steinmetz and Wright (152) clearly demonstrated that $C$. zizaniae and not $C$. purpurea is the pathogen of Zizania. In our examination of collections of Claviceps from Zizania in ND, ME, and $\mathrm{MN}$, the oval-elongate conidia were typical for $C$. zizaniae and considerably larger than conidia of $C$. purpurea var. purpurea. In addition, the less dense, more elliptically shaped sclerotia of $C$. zizaniae clearly differ from the generally tapered sclerotia of $C$. purpurea var. purpurea. 
Table 3. List of grasses reported as hosts of Claviceps purpurea in the United States, based on published reports, inclusion in the National Fungus Collection database, or other herbaria, after examination and confirmation of host ${ }^{\mathrm{a}}$

\begin{tabular}{|c|c|}
\hline Host & Source \\
\hline \multicolumn{2}{|l|}{ Arundinoideae } \\
\hline \multicolumn{2}{|l|}{ Arundineae } \\
\hline $\begin{array}{l}\text { Phragmites australis (Cav.) Trin. ex Steud. } \\
=\text { Phragmites phragmites }(\text { L.) H. Karst } \\
\text { = Phragmites communis Trin. }\end{array}$ & $\begin{array}{l}\text { DC (BPI); IA (ISC, } 9,53,116,136) ; \mathrm{NJ}(9,136) ; \mathrm{ND}(136,162) ; \mathrm{OK}(9, \\
\quad 41,126,136)\end{array}$ \\
\hline \multicolumn{2}{|l|}{ Danthonieae } \\
\hline Danthonia paryii Scribn. & $\mathrm{CO}(136)$ \\
\hline Danthonia spicata (L.) Beauv. ex Roemer \& Schult. & MI (MICH, 9, 123, 136); WI (BPI) \\
\hline Molinia caerulea $($ L.) Meonch & $\mathrm{DC}(\mathrm{BPI})$ \\
\hline \multicolumn{2}{|l|}{ Stipeae } \\
\hline $\begin{array}{l}\text { Achnatherum lemmonii (Vasey) Barkworth } \\
\text { = Stipa columbiana Macoun }\end{array}$ & ID (WSP, 136); IN (9); UT (BPI, WSP, 9, 136); WA (9) \\
\hline $\begin{array}{l}\text { Achnatherum lettermanii (Vasey) Barkworth } \\
\text { = Stipa lettermanii Vasey }\end{array}$ & WA (WSP, 9, 136, 147); WY (RM) \\
\hline $\begin{array}{l}\text { Achnatherum nelsonii (Scribn.) Barkworth } \\
\text { = Stipa columbiana var nelsonii (Scribn.)Hitchc. }\end{array}$ & UT (BPI); WA $(136,148)$ \\
\hline $\begin{array}{l}\text { Achnatherum nelsonii (Scribn.) Barkworth ssp. dorei (Barkworth \& J.R. } \\
\text { Maze) Barkworth } \\
\text { = Stipa minor (Vasey) Scribn. }\end{array}$ & IN (105) \\
\hline $\begin{array}{l}\text { Achnatherum robustum (Vasey) Barkworth } \\
\text { = Stipa robusta (Vasey) Scribn. }\end{array}$ & MT $(99,136)$ \\
\hline $\begin{array}{l}\text { Achnatherum scribneri (Vasey) Barkworth } \\
\text { = Stipa scribneri Vasey }\end{array}$ & $\mathrm{CO}(\mathrm{NY})$ \\
\hline $\begin{array}{l}\text { Heterostipa comata (Trin. \& Rupr.) Barkworth } \\
=\text { Stipa comata Trin. \& Rupr. }\end{array}$ & MT (MONTU); ND $(136,162)$ \\
\hline $\begin{array}{l}\text { Heterostipa spartae (Trin.) Barkworth } \\
=\text { Stipa spartea Trin. }\end{array}$ & ND $(136,162) ;$ WI (WIS, 63) \\
\hline $\begin{array}{l}\text { Nassella viridula (Trin.) Barkworth } \\
=\text { Stipa viridula Trin. }\end{array}$ & $\begin{array}{l}\text { IA (ICS, 52, 157); MN (9, 45, 127, 136); ND (BPI, 9, 44, 136, 162); SD } \\
\quad(106)\end{array}$ \\
\hline Oryzopsis asperfolia Michx. & NY (CUP) \\
\hline Oryzopsis racemosa (Sm.) Ricker ex A.S. Hitchc. & WI (61) \\
\hline $\begin{array}{l}\text { Piptatherum miliaceum (L.) Cosson } \\
\text { = Oryzopsis milacea (L.) Asch. \& Schweinf. }\end{array}$ & $\mathrm{DC}(\mathrm{BPI})$ \\
\hline \multicolumn{2}{|l|}{ Chloridoideae } \\
\hline Bouteloua curtipendula (Michx.) A. Gray & $\mathrm{NE}(9,136) ; \mathrm{OK}(136)$ \\
\hline Bouteloua gracilis (Kunth) Lag. ex. Steud. & $\mathrm{OK}(125,136)$ \\
\hline Buchloe dactyloides (Nutt.) Engelm. & $\mathrm{OK}(\mathrm{BPI})$ \\
\hline Cynodon dactylon (L.) Pers. & OK $(25,26)$ \\
\hline Distichlis spicata (L.) Greene & DC (BPI); MD (136); MS (9, 118); WA (FH, UC, 9, 136) \\
\hline \multicolumn{2}{|l|}{ Pooideae } \\
\hline \multicolumn{2}{|l|}{ Aveneae } \\
\hline Agrostis canina $\mathrm{L}$. & IA (ICS, 52, 157) \\
\hline $\begin{array}{l}\text { Agrostis capillaris } \mathrm{L} . \\
=\text { Agrostis tenuis } \text { Sibth. } \\
=\text { Agrostis vulgaris With. }\end{array}$ & DC (BPI); IA (116); OR (BPI, 2, 9, 135, 136); WA (BPI, 17, 136); WI (WIS) \\
\hline Agrostis exarata Trin. & AK $(22,136) ;$ OR (BPI, NY, OSC, 9, 135, 136); UT (NY) \\
\hline Agrostis hyemalis (Walter) B.S.P. & ND $(9,162) ;$ OK $(9,41) ;$ WI $(9,61)$ \\
\hline Agrostis scabra Willd. & ND (136); OK $(125,136) ; \mathrm{WI}(\mathrm{BPI}, 136)$ \\
\hline $\begin{array}{l}\text { Agrostis stolonifera } \mathrm{L} \text {. } \\
=\text { Agrostis alba } \mathrm{L} . \\
=\text { Agrostis alba } \text { var. ovularies } \\
=\text { Agrostis palustris } \text { Huds. }\end{array}$ & $\begin{array}{l}\text { CO (BPI); DC (BPI); IA (ICS, 9, 53, 136, 157); IL (ILLS, 16, 74, 136); MI } \\
\text { (BPI, MSC, 9, 123); MN (127, 136); ND (9, 136, 162); OH (BPI, 9, 76); } \\
\text { OR (BPI, OSC, NY, WSP, 2, 9, 135, 136); PA (9); SD (FH); WI }(45,149) \text {; } \\
\text { PA to IA (9) }\end{array}$ \\
\hline $\begin{array}{l}\text { Alopecurus borealis Trin. } \\
=\text { Alopecurus alpinus } \text { Sm. } \\
=\text { Alopecurus occidentalis Scribn. \& Tweedy }\end{array}$ & MT (BPI); WY (BPI) \\
\hline Alopecurus geniculatus L. & ND (RM, 9, 136, 162) \\
\hline Alopecurus pratensis $\mathrm{L}$. & $\begin{array}{l}\text { IN }(9,105) ; \text { IA (ICS, 52, 157); MI (MSC, 9); MT (BPI; 9, 44, 136); ND } \\
(136,162) \text {; OR (BPI, NY, 9, 44, 135, 136); SD (106) }\end{array}$ \\
\hline Ammophila arenaria (L.) Link & MI (BPI, MICH); OH (NY); WI (BPI, WIS, 136) \\
\hline Ammophila breviligulata Fern. & IN (9); MI (BPI, 9; 105); NY (BPI, NY); NC (54); OH (9); WI (WIS, 61) \\
\hline Anthoxanthum odoratum $\mathrm{L}$. & NY (NY) \\
\hline Arctagrostis latifolia (R. Br.) Griseb. & AK (BPI, 22) \\
\hline Arrenatherum elatius (L.) J. Presl \& C. Presl & $\begin{array}{l}\text { IN }(9,105) \text {; IA (ICS, 52, 157); KY }(9,73) \text {; MO (BPI, 9, 99, 136); MT }(9 \text {, } \\
\text { 44, 136); NE (BPI); OH }(9,99,136) \text {; OR }(9,44,135,136) \text {; WI }(9,45,61 \\
\text { 136); WV (BPI, 39) }\end{array}$ \\
\hline $\begin{array}{l}\text { Arrenatherum elatius var. bulbosum (Willd.) Spenner } \\
\text { = Arrhenatherum tuberosum (Gilib.) F.W. Schultz }\end{array}$ & IA $(52,157)$ \\
\hline Avena sativa $\mathrm{L}$. & $\begin{array}{l}\mathrm{CO}(9,136) ; \mathrm{DC}(\mathrm{BPI}) ; \mathrm{IL}(16) ; \mathrm{IA}(\mathrm{BPI}, 9,52,53,136) ; \mathrm{KS}(9,108) ; \mathrm{MI} \\
\text { (9); MN }(9,85,127) ; \mathrm{NY}(9) ; \mathrm{ND}(9,85,108,136,162) ; \mathrm{OH}(37) ; \mathrm{OK}(9 \text {, } \\
\text { 41); SD }(106) \text {; WA }(9,75,136) ; \text { scattered in east }(136) .\end{array}$ \\
\hline
\end{tabular}

\footnotetext{
${ }^{\text {a }}$ Herbaria were inventoried for ergot during 1996 to 1999. Herbarium codes are those listed in Index Herbariorum (81).

${ }^{\mathrm{b}}$ Most reports of Sprague et al. prior to 1958 of fungi on E. condensatus belong under E. cinereus (139).
} 
Table 3. (Continued from previous page)

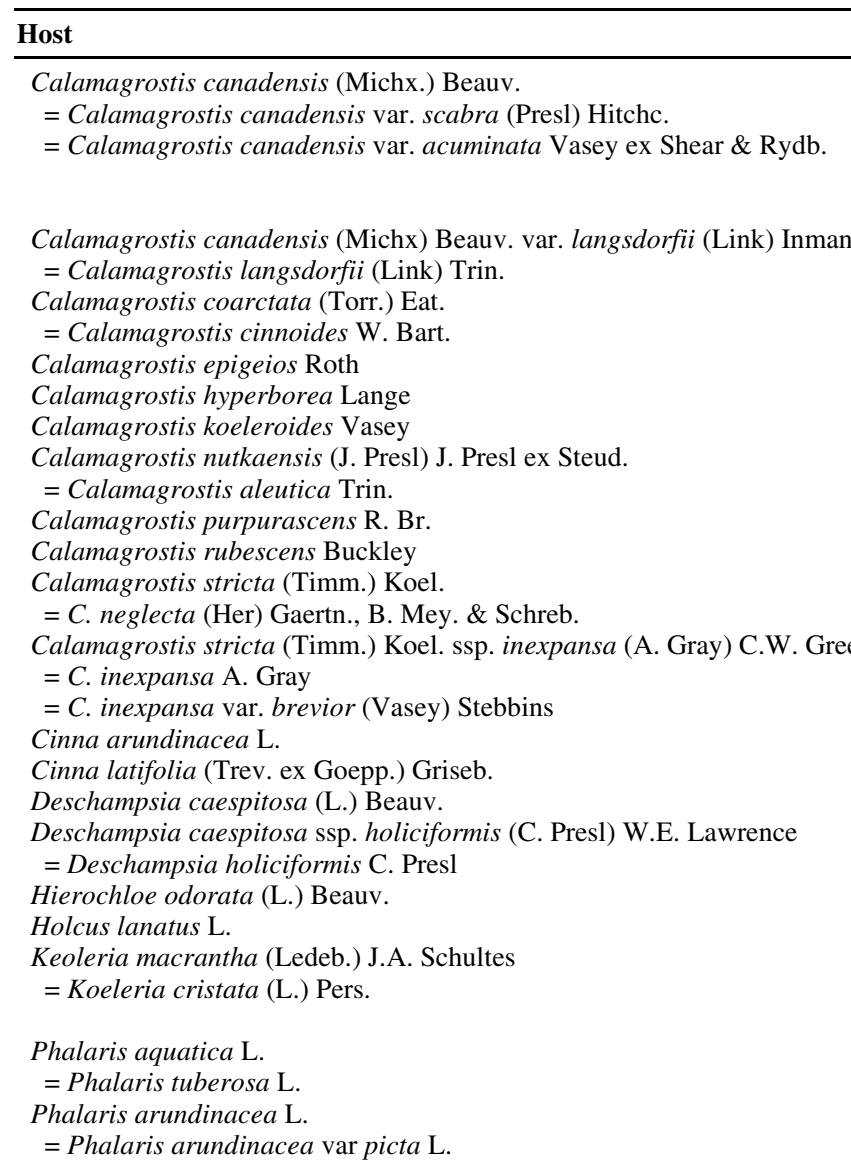

Phalaris californica Hook. \& Arn.

Phalaris canariensis L.

Phalaris caroliniana Walt.

Phleum pratense $\mathrm{L}$.

Trisetum canescens Buckley

Bromeae

Bromus anomalus Rupr. ex Fourn.

Bromus carinatus Hook. \& Arn.

= Bromus marginatus Nees ex Steud.

= Bromus polyanthus Scribn.

Bromus ciliatus L.

Bromus commutatus Schrad.

Bromus erectus Huds.

Bromus erectus subsp. condensatus (Hack.) Ascher. \& Graebn.

= Bromus condensatus Hack.

Bromus inermis Leyss.

Bromus inermis Leyss. var. pumpellianus (Scribn.) C.L. Hitchc.

= Bromus pumpellianus Scribn.

Bromus japonicus Thunb. ex Murray
Source

AK (BPI, WIS, WSP, 136); IL (BPI); IA (ICS, 53, 115, 116, 136, 157, 158);

ME (BPI, CUP, 9, 136); MA (FH); MI (BPI, 9, 136); MN (BPI, MSC,

WIS, 45, 85, 127, 136, 149); MT (FH); NH (BPI); NY (BPI, CUP); OR (136); SD (106, 164); WA (UC, 9, 136); WI (BPI, WIS, 45, 60, 61, 136); $\mathrm{ME}$ to $\mathrm{MN}(9)$

AK (BPI, 22)

MA (BPI)

IA (ICS, 52, 157, 165)

ND (162)

$\mathrm{CA}(\mathrm{UC})$

AK (BPI, 22, 136); OR $(135,136)$

$\mathrm{CO}(\mathrm{BPI})$

MT (WSP, 138)

MN (75, 127); NE (NEB); ND (BPI, OSC, NY, 19, 136); WI $(56,61,136)$

MN (WSP, 9, 127, 142); MT (9, 136, 147); ND (OSC, 9, 44, 136); SD (FH, 106); WI (WIS, 66).

WI (WIS, WSP, 61, 136)

AK (144)

IA (ICS, 52, 157); OR $(9,44,136)$

DC (BPI); WA (WIS)

CT (ICS); ND $(136,162)$

OR (BPI, OSC, NY, 9, 135, 136); WA (BPI, CUP, WIS, WSP, 9).

AZ (BPI, WIS, 9, 136); ID (WSP, 9, 136, 147); IA (BPI, 9, 53, 136); KS (9, 136); MN (127); MO (136); MT (WSP, 6, 24, 136); ND (136, 162); OK $(9,41,126,136)$; UT (10); WI (BPI, WIS, 9, 60, 61, 136)

IA (ICS)

CA (12); CT (BPI, WIS); DE (NY); IL (BPI, 99, 136); IN (MICH); IA (ICS, 52, 157, 158); KS (BPI); ME (BPI, 136); MD (BPI); MA (BPI, GH, NY); MI (MICH); MN (85, 127, 136); MT (6, 9, 24, 136); NY (BPI, CUP, WIS 9, 136); ND (136, 162); OK (9, 41, 126, 136); PA (BPI, 76, 79, 93, 108); SD (106, 164); VA (BPI, CUP, FH, 136); WI (BPI, WIS, 5, 60, 61, 136); ME to VA $(9,136)$;

IA (ICS, 52, 157)

IA $(52,157)$; MI $(9,123,136) ; \operatorname{MN}(9,127,136)$

IA (ICS, 52, 58)

AK (BPI; 9, 22, 136); CT (BPI, 136); DC (BPI); IL (BPI, F, NY); IN (MIN, 105); IA (BPI, 53, 113, 116, 136, 157); KY (9, BPI); ME (9, BPI); MI (BPI, MSC, NY, 123); MN (BPI, 9, 45, 85, 127, 136, 155); NY (NY); ND (136, 162); OK (41); OR (BPI, OSC, 9, 135, 136); PA (BPI); SD (NY, 106); TX (BPI); WA (9); WI (BPI, CUP, UC, WSP, 45, 61, 116, 136); ME to KY (9); scattered in the east (136)

ID (WSP, 9, 148, 136)

WY (RM)

CA (BPI, F, WIS); ID (WSP, 44, 136); IN (105, 136); IA (ICS, 52, 107 157); KY (73); MT (9, 44, 136); NV (9); NH (BPI); OR (WSP, 135, 136); UT (RM, NY, 136); WA (WSP, 9, 44, 136); WY (BPI, RM, 136, 147); MT to NV (9)

MT $(9,44,136)$; WI (WIS, 61, 136)

IL (ILLS); IA $(52,157)$

DC (BPI); MN (9, 99, 127, 136); MT (9, 44, 136); NY (BPI, 9) ID (45)

AZ (136); CO (15, 136); DC (BPI, 136); ID (BPI, WSP, 136, 147); IL (ILLS, 16, 99, 136); IN (BPI, 105); IA (BPI, 53, 86, 107, 108, 136, 157); KS (BPI, 86, 131); KY (72, 73); MD (136); MA (NY); MI (MSC, 13, 71, 136); MN (BPI, WIS, NY, 45, 56, 75, 99, 127, 136, 155); MO (BPI, 99, 136); MT (WSP, 99, 136); NE (BPI, NEB, 1, 86, 136); NH (92); NY (BPI); ND (BPI, WSP, 19, 44, 75, 99, 136, 162); OK (41); SD (BPI, NY; 44, 106, 136); UT (BPI); WA (BPI, WIS, WSP, 42, 136); WV (BPI, 38); WI (BPI, WIS, 5, 45, 56, 60, 61 82, 99, 136); WY (BPI, 14, 136); widespread (9); general eastward (136) AK (WIS); MN (127, 136); MT (136)

IA $(52,107,157)$ 
Table 3. (Continued from previous page)

\begin{tabular}{|c|c|}
\hline Host & Source \\
\hline $\begin{array}{l}\text { Bromus kalmii Gray } \\
=\text { Bromus purgans L. }\end{array}$ & IA (ICS, 52,107, 157) \\
\hline Bromus secalinus L. & $\begin{array}{l}\text { IL (ILLS); IA (ICS, 52, 157); IN (9, 105, 136); KY (9, 73); MO (F); WA } \\
\text { (WSP, 136, 148); WV (BPI, 38) }\end{array}$ \\
\hline Bromus tectorum $\mathrm{L}$. & $\mathrm{KS}(\mathrm{BPI})$ \\
\hline \multicolumn{2}{|l|}{ Meliceae } \\
\hline Glyceria borealis (Nash) Batch. & $\begin{array}{l}\text { ID (BPI, 136); ME (BPI, 136); MI (MICH, 136); NY (CUP); WI (BPI, F, } \\
\text { 32, 60, 61, 136) }\end{array}$ \\
\hline Glyceria canadensis (Michx.) Trin. & ME (BPI, 136) \\
\hline $\begin{array}{l}\text { Glyceria fluitans (L.) R. Br. } \\
=\text { Panicularia fluitans (L.) Kuntze }\end{array}$ & IA $(53,115,116) ;$ WI (WIS, 9, 45) \\
\hline $\begin{array}{l}\text { Glyceria grandis S. Watson ex A. Gray } \\
=\text { Panicularia grandis (S. Wats.) Nash }\end{array}$ & IA (136); ND (136, 162); WI (63) \\
\hline Glyceria septentrionalis A. S. Hitchc. & IA (ISC, 9); IN (9, 136); WI $(9,61,136)$ \\
\hline $\begin{array}{l}\text { Glyceria striata (Lam) A.S. Hitchc. } \\
=\text { Glyceria nervata (Willd.) Trin. }\end{array}$ & OK $(9,41,126,136) ;$ WA $(9,136) ;$ WI (BPI, 9, 61, 136) \\
\hline \multicolumn{2}{|l|}{ Poeae } \\
\hline Arctophila fulva (Trin.) Rupr. ex Andress. & $\mathrm{AK}(22,136)$ \\
\hline Briza media $\mathrm{L}$. & $\mathrm{DC}(\mathrm{BPI})$ \\
\hline Dactylis glomerata L. & $\begin{array}{l}\text { AK (BPI, 22, 136); AZ (136); CA (12); CT (136); IL (ILLS); IN (BPI, 105); } \\
\text { IA (BPI, ICS, 52, 107, 113, 157); KS (BPI); KY (73, 99, 136); ME (BPI, } \\
\text { 9, 136); MD (84); MI (MSC, 70, 71, 136); MN (45, 127, 136); MT (99, } \\
\text { 136); NH (92); NY (BPI, CUP, MIN, 163); ND (162); OK (9, 41, 126, } \\
\text { 136); OR (BPI, 2, 44, 135, 136); TN (BPI, 99, 136); VA (9); WA (UC, } \\
\text { WIS, WSP, 9, 136);WV (BPI); WI (BPI, WIS, 5, 45, 60, 61, 136); ME to } \\
\text { VA (9); general in the east (136) }\end{array}$ \\
\hline $\begin{array}{l}\text { Festuca arvernensis Auquier, Kerguelen \& Markgr.-Dannenb. } \\
\text { = Festuca ovina var. glauca (Lam.) W.D.J. Koch }\end{array}$ & MO (99) \\
\hline $\begin{array}{l}\text { Festuca heterophylla Lam. } \\
\text { = Festuca rubra } \text { var. heterophylla (Lam.) Mutel }\end{array}$ & $\mathrm{MN}(45,85,127)$ \\
\hline Festuca idahoensis Elmer & $\begin{array}{l}\text { ID }(9,44,148,136) ; \text { MT (WSP, 136); ND (136); OR (WSP, 9, 148, 136); } \\
\text { WY (BPI) }\end{array}$ \\
\hline $\begin{array}{l}\text { Festuca kingii (S. Wats.) Cassidy } \\
=\text { Festuca confinis Vasey }\end{array}$ & CO (136, 147); UT (BPI); WY (WSP, 136, 147) \\
\hline Festuca ovina L. & $\begin{array}{l}\text { AK (WIS); ID (140); IA (ICS, 52, 157); MD (BPI, 9); MO (9, 136); WI } \\
\text { (BPI, WSP, 61, 136) }\end{array}$ \\
\hline Festuca rubra L. & $\begin{array}{l}\text { AK (BPI, 22); DC (BPI); IA (52, 157); KY }(9,136) ; \text { MA (WIS); MN (9, } \\
\text { 127, 136); NY (9, 136); ND (BPI, 136); OR }(44,136) ; \text { SD (106); WA (44, } \\
136) ; \text { NY to KY and MN and Pacific Northwest }(9)\end{array}$ \\
\hline $\begin{array}{l}\text { Festuca rubra ssp. falax Thuill. } \\
\text { = Festuca rubra } \text { var. commutata } \text { Gaud. }\end{array}$ & KY $(73,136) ;$ ND (136); OR (2); WA $(44,136)$ \\
\hline $\begin{array}{l}\text { Festuca subverticillata (Pers.) Alexeev. } \\
=\text { Festuca obtusa } \text { Biehler }\end{array}$ & WI (WIS; 65) \\
\hline Lolium arundinaceum (Schreber) Darbysh & CA (12); CT (BPI); FL (4): IL (ILLS); IN (105); IA (ICS, 52, 157); KY (72, \\
\hline $\begin{array}{l}=\text { Festuca arundinacea } \text { Schreb. } \\
=\text { Festuca elatior } \mathrm{L} .\end{array}$ & $\begin{array}{l}\text { 73, 136); ME (BPI, 136); MD (BPI); MA (BPI); MN }(45,127) \text {; NY (BPI; } \\
\text { CUP, 75, 163); NC (54); ND (136, 162); OK }(41,126,136) ; \text { OR }(2,44\end{array}$ \\
\hline = Festuca elatior var. arundinacea (Schreb.) Wimm. & $\begin{array}{l}135,136) ; \text { PA (BPI, 108); VT (BPI); VA (BPI, CUP, 136); WA (WSP, } \\
136,145) ; \text { WV (BPI); WI (WIS, 45, 64); general (9); ME to VA (136) }\end{array}$ \\
\hline $\begin{array}{l}\text { Lolium giganteum (L.) Darbysh. } \\
=\text { Festuca gigantea }(\text { L.) Vill. }\end{array}$ & DC (BPI) \\
\hline $\begin{array}{l}\text { Lolium multiflorum Lam. } \\
=\text { Lolium italicum A. Braun }\end{array}$ & $\begin{array}{l}\text { CA }(9,44,136) ; \text { KY }(9,72,73,136) \text {; OK }(9,41,125,136) \text {; OR (BPI, OSC, } \\
\text { WSP, 9, 44, 135, 136); WA (BPI, OSC, WSP, 9, 93, 136) }\end{array}$ \\
\hline Lolium perenne $\mathrm{L}$. & $\begin{array}{l}\text { CA (BPI, 9, 44, 136); CT (BPI); DC (BPI, WIS, WSP); ID }(9,136) \text {; IL } \\
\text { (ILLS); IA }(52,157) ; \text { KS }(9,136) ; \text { MD (BPI, 9, 136); MA (NY); MI }(9 \\
\text { 71, 136); MN }(9,127,136) ; \text { NE }(9,136) ; \text { ND }(136,162) ; \text { OK }(9,41,125 \\
\text { 136); OR (OSC, 2, 9, 44, 135, 136); PA (BPI); SD (9, 136); VA }(9,136) ; \\
\text { WA (BPI, WSP, 9, 93); WV (BPI, 9, 136); WI (BPI, WIS, 59, 61, 136); } \\
\text { WY (NY) }\end{array}$ \\
\hline $\begin{array}{l}\text { Lolium pratense (Hudson) Darbysh. } \\
=\text { Festuca pratensis Huds. }\end{array}$ & DC (BPI); MA (NY); MN (45); VT (BPI) \\
\hline $\begin{array}{l}\text { Lolium rigidum ssp. lepturoides (Boiss.) Sennen \& Maurico } \\
=\text { Lolium subulatum Vis. }\end{array}$ & CA (WSP, 140) \\
\hline Poa anпua $\mathrm{L}$. & IA (116); MO (136); OK $(9,41,126,136)$ \\
\hline Poa arida Vasey & $\mathrm{ND}(9,136,148)$ \\
\hline Poa compressa $\mathrm{L}$. & $\begin{array}{l}\text { IL (ILLS, 16); IN (105); IA (ICS, 52, 157); KY (9); MN }(85,127,136) ; \text { MO } \\
\text { (136); MT (9, 99, 136); ND (136, 162); OK }(9,41,124,126,136) ; \text { SD } \\
\text { (164); WA (WSP, 9, 136, 148); WI (BPI, 45, 61, 136); northeastern and } \\
\text { north central states to KY (9) }\end{array}$ \\
\hline $\begin{array}{l}\text { Poa fendleriana (Steud.) Vasey } \\
=\text { Poa longiligula } \text { Scribn. \& Williams }\end{array}$ & CO (9); ND (9); UT (BPI, NY, 136); WA (9); ND to CO (9) \\
\hline Poa nemoralis L. & WA $(44,136)$ \\
\hline Poa nervosa (Hook.) Vasey & WY (136) \\
\hline Poa palustris L. & IN (105); MN (127, 136); ND (BPI, 9, 44, 134, 136); SD (106, 136) \\
\hline & (Continued on next page) \\
\hline
\end{tabular}


Table 3. (Continued from previous page)

\begin{tabular}{|c|c|}
\hline Host & Source \\
\hline Poa pratensis L. & $\begin{array}{l}\text { AK (WSP, 143); IL (BPI); KY }(9,73,99,136) \text {; IA (BPI, 85, 113, 116, 136, } \\
\text { 157); MI (BPI, MSC); MN (45, 85, 127, 136); MO (BPI); MT }(9,44,136) \text {; } \\
\text { NY (136); ND (136, 162); OH (136); OK }(9,41,126,136) \text {; OR (OSC, 2); } \\
\text { PA (136); SD (164); UT (FH, 136); WA (136); WI }(5,45,61,82,136) ; \\
\text { WY (WSP, 136); north eastern and north central states to KY (9) }\end{array}$ \\
\hline Poa pratense L. $\times$ Poa arachnifera Torr. & IA $(52,157) ; \mathrm{MO}(\mathrm{BPI}, 99,136)$ \\
\hline $\begin{array}{l}\text { Poa secunda } \text { J. Presl } \\
=\text { Poa canbyii } \text { Vasey } \\
=\text { Poa gracillima } \text { Vasey } \\
=\text { Poa iuncifolia } \text { Scribn. }\end{array}$ & $\begin{array}{l}\text { CO (9); ID (WSP, 136, 147, 148); MT }(99,136) \text {; ND }(9,134,136) \text {; OR } \\
\text { (BPI, WSP, 135, 136, 145); UT (WSP, 136, 145); WA (WSP, 9, 43, 44, } \\
\text { 136); WY (BPI, 136); ND to CO (9) }\end{array}$ \\
\hline
\end{tabular}

juncifolia Scribn.

= Poa longifolia Trin.

= Poa nevadensis Vasey

= Poa scabrella (Thurb.) Benth.

Poa secunda J. Presl ssp. nevadensis (Scribn.) Soreng

= Poa ampla Merr.

Poa stenantha Trin.

Poa tibetica Munro ex Stapf.

Puccinellia nutkaensis (J. Presl) Fern. \& Weatherby

Pucinellia distans (Jacq.) Parl.

Torreyochloa pallida (Torr.) Church var. pauciflora (J. Presl) J.I. Davis

= Glyceria pauciflora Presl

Vulpia octoflora (Walt.) Rydb.

= Festuca octoflora Walt.

Triticeae

Agropyron cristatum (L.) Gaertn.

Agropyron desertorum (Fisch. ex Link) J.A. Schult.

Agropyron fragile (Roth) Candargy ssp. sibiricum (Willd.) Melderis

= Agropyron sibiricum (Willd.) P. Beauv.

Agropyron michnoi Roshev.

Agropyron trachycaulum ssp. subsecundus (Link) A. \& D. Love

= Agropyron trachycaulum (Link) Malte var. glaucum (Pease \& Moore)

Malte

Elyhordeum $\times$ Agrohordeum macounii (Vasey) Lepage

= Elymus macounii Vasey

Elymus $\times$ Pseudorepens (Scribn. \& J.G. Sm.) Barksworth \& Dewey

= Agropyron pseudorepens

Elymus alaskanus (Scribn. \& Merr.) A. Love ssp. alaskanus

= Agropyron violaceum (Hornem.) Lange

Elymus canadensis L.

$=$ E. canadensis var. robustus (Scribn. \& J.G. Sm.) Mackenzie \& Bush

$=$ E. robustus Scribn. \& Smith

Elymus caninus (L.) L.

= Agropyron caninum (L.) Beauv.

Elymus dahuricus Turcz. ex Griseb.

Elymus dahuricus Turcz. ex Griseb. ssp. excelsus (Turcz. ex Griseb.) Tzvel.

= Elymus excelsus Turcz. ex Griseb.

Elymus elymoides (Raf.) Swezey

= Sitanion hystrix (Nutt) J.G. Sm.

$=E$. sitanion Schultes

Elymus glaucus Buckley

Elymus glaucus ssp. virescens (Piper) Gould

$=$ E. virescens Piper

Elymus hystrix L.

= Hystrix hystrix (L.) Millsp.

= Hystrix patula Meonch

= Asprilla hystrix Humb.

Elymus interruptus Buckl.

Elymus lanceolatus (Scribn. \& J.G. Sm.) Gould

= Agropyron dasystachyum (Hook.) Scribn.

= Agropyron riparium Scribn. \& J.G. Smith

Elymus lanceolatus (Scribn. \& J.G. Sm.) Gould ssp. albicans (Scribn. \&

J.G. Sm.) Barkworth \& Dewey

= Agropyron albicans Scribn. \& J.G. Sm.

DC (BPI); ID (44, 136); MT (99, 136); NC (BPI); ND (134, 136, 148); WA $(42,43,44,136)$

WA $(44,136)$

DC (BPI)

AK (WSP, 143)

WA $(9,44,136)$

AK $(22,136,143)$

MI $(9,136)$

DC (BPI); IN (105, 136); IA (ICS, 52, 157); KY (73); MA (BPI); MI (71,

136); MN (85, 127, 136); MT (BPI, WSP, 44, 99, 136); ND (BPI, 44,

136); OR (BPI, NY, 135, 136); SD (44, 99, 106, 136); WA (BPI, NY, 44, 136); WY (WIS, 14, 136); general (9)

MD (BPI); MI (71, 136); MN (85, 127); ND (136); SD (106); WA (136, 146); WY (RM; 50)

DC (BPI); MI (70, 71, 136); MN (127, 136); MT (BPI; 136); ND (BPI, 44, 136); general (9)

DC (BPI); ND (136)

WI (BPI, 57, 61)

IA (BPI); MN (75, 127); MT (BPI, 44, 136); NE (NEB, 18); ND (BPI, 44, 136, 162); SD (BPI, 106); WA (WSP, 136, 145)

NE (NEB); WY (BPI)

$\operatorname{MT}(7)$

CO (BPI); ID (136); IL (BPI, ILLS); IA (BPI, WIS, 8, 53, 116, 136, 158); KS (BPI, FH, NY, 89, 131); MI (71); MN (45, 127, 136); MO (BPI, 136); MT (ICS); NE (BPI, NEB, 1, 136); NM (BPI, 136, 150); ND (136, 162); OH (NY); OK (41, 97, 125, 136); OR (OSC, 135, 136); SD (BPI, NY, 102, 106, 136, 164); TX (BPI, 23, 136); UT (BPI, 136); WA (136); WI (BPI, WIS, 43, 45, 136); WY (BPI, 14, 136, 151). general in the east (136); general (9)

IA (BPI, ISC, 53); MI (MSC, 70, 71, 136); MN (45, 85); ND (136); SD

(NEB, 164); WA (44, 136); WI (45)

ND $(9,148,136)$; WA $(9,44,136)$

WA $(9,44)$

AZ (BPI); MT (BPI); OR (WSP, 9, 44, 136)

CA (BPI; 136); ID (136, 147); IA (BPI, 52, 157); MI (MICH); MT (WSP); ND (44); OR (BPI, OSC, WSP, 44, 80, 135, 136); WA (44, 136); WY (BPI, RM, UC, 50, 136); general (9)

IN (105)

IA (ISC, 9, 53, 115, 116, 136); IN (9, 105, 136); MN (127); ND (136, 162); WI (ICS)

TN (BPI)

ID (WSP, 136, 147); MT (44, 99, 136); ND (44, 136, 148); SD (106); WA (44, 136); WY (NY, UC, 50, 136); Northern Great Plains and Pacific Northwest (9)

ND (136)

(Continued on next page) 
Table 3. (Continued from previous page)

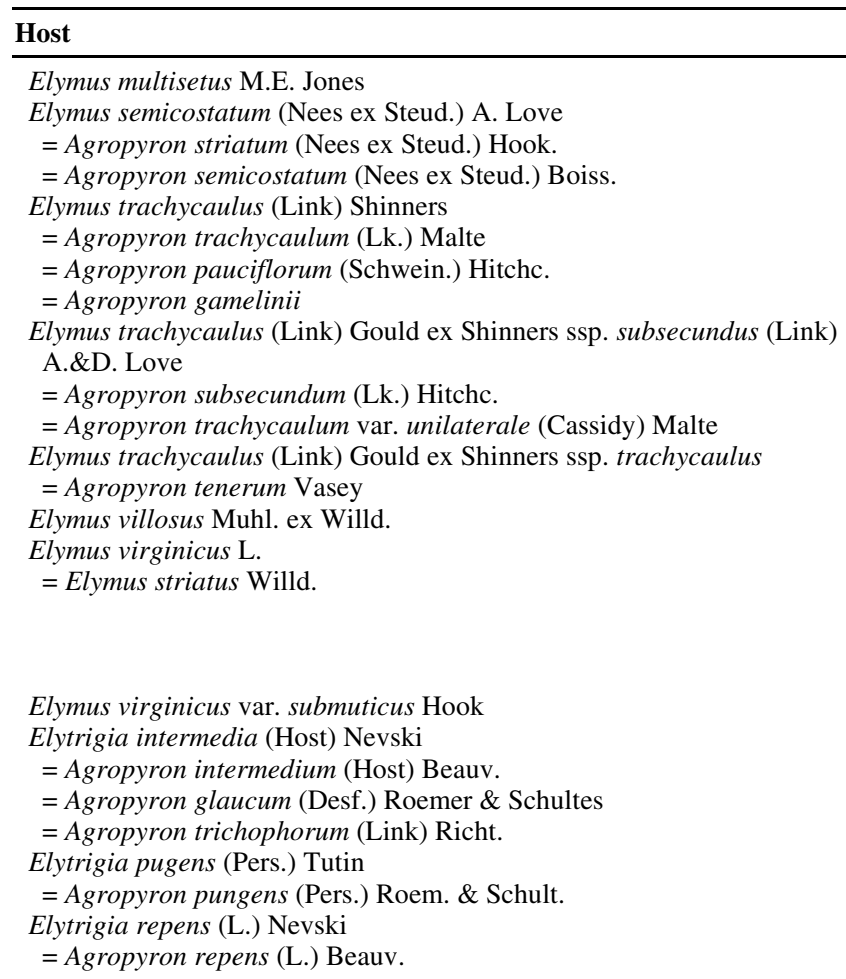

Eremopyrum triticeum (Gaertn.) Nevski

= Agropyron triticum Gaertn.

Hordeum brachantherum Nevski

= Hordeum nodosum $\mathrm{L}$.

= Hordeum nodosum var. boreale (Scribn. \& Sm.) Hitchc.

= Hordeum boreale Scribn \& J.G. Sm.

Hordeum jubatum L.

= Hordeum jubatum var. caespitosum (Scribn. ex Pammel) A.S. Hitchc.

Hordeum murinum L.

Hordeum vulgare L.

\section{Source}

OR (WSP)

MI (71, 136); ND (136); WA (136)

AK (137); AZ (136); ID (136); IA (ICS, 52, 136, 157, 158); MI (MSC), MN (BPI, 127); MT (44, 99, 136, 147); NM (136); ND (44, 136); OR (44, 135, 136); SD (BPI; 99, 106, 136); UT (BPI, 136, 147); WA (44); WI (136); WY (BPI, WSP, 50, 136); general (9)

ID (136, 147); IA (WSP, 136); MI (136); MN (136); MT (WSP, 136, 147); SD (136); UT (BPI, WSP, 136); WA (44, 136); WI (UC, 61); general (9)

IA (53); MI (MICH); MN (45, 85); NM (BPI); ND (162); SD (BPI; 164); WI (45)

IA (ICS); WI (61)

IL (BPI, ILLS); IN (105); IA (BPI, UC, 53, 115, 116, 136, 157); KS (BPI, FH, NY, 131, 136); LA (BPI); MI (71, 136); MN (45, 85, 127); MO (BPI, 99, 136); NE (BPI, 1, 136); NY (CUP); ND (BPI, 19, 136, 162); OK (BPI, 41, 97, 125, 136); SD (NEB, 106); TX (BPI, 23, 136); WI (BPI, 45, 63); general (9); general eastward (136)

KS (116)

IA (ICS, 52, 115, 157); KS (BPI); KY (73); MN (85, 127); MT (BPI); NE (BPI); ND (136); SD $(106,136,164) ;$ WA $(44,136)$

MT (99); WA (BPI)

CT (BPI); DC (BPI); IL (BPI, ILLS); IN (105); IA (BPI, 53, 107, 108, 113 , $115,116,136,157,158,165) ; \mathrm{KS}$ (136); KY (73); ME (BPI, FH); MD (BPI, 78, 79); MA (BPI, NY, 99, 136); MI (MSC, 13, 71, 75, 136); MN (BPI, 45, 74, 75, 85, 99, 127, 136, 155); MO (136); MT (WSP, 136); NE (BPI, NEB, 74,136); NH (BPI, WIS); NY (BPI, 75, 78, 99, 136); ND (BPI, 19, 44, 136, 162); OH (BPI, 136); OK (UC, 41, 125, 136, 163); OR (BPI, NY, 135, 136); PA (BPI, WSP, 76, 79, 94, 108); SD (BPI, 44, 106, 136); VT (BPI, FLAS); VA (BPI); WA (136, 148); WV (BPI, 38, 136); WI (BPI, WIS, 5, 45, 60, 61, 82, 99, 136); general (9). WY (BPI)

AK (BPI, 9, 22, 136); CO (136); MN (9, 45, 127, 136, 148); WA (136)

IA (BPI, 9, 136); MT (9, 44, 136); NE (129); ND (136, 162); OK (9, 41, $126,136)$; OR $(9,44,136)$; WA (BPI, 9,136$)$; WI $(9,45,136)$

WA (9)

CO (15, 108, 136); DC (BPI); ID (78, 136); IL (77, 108); IN (74, 79, 85, 93); IA (BPI, 45, 53, 79, 85, 108, 113, 125, 149); MA (79); MI (74, 79); MN (BPI, 45, 95, 77, 78, 79, 85, 108, 127, 136, 149, 155); MO (85, 136); MT (136, 149); NE (BPI, 1, 77, 129); ND (BPI, 13, 77, 79, 93, 108, 136, 149); OH (79); OK (41, 125, 136); OR (135, 136); SD (106); VA (130); WA (136); WI (45, 74, 79, 85, 93, 108, 113, 149); WY (130, 136, 151), general (9), general in east (136)

MT $(44,136)$

Leymus ambiguus (Vasey \& Scribn.) Dewey

= Elymus ambiguus Vasey \& Scribn.

Leymus angustus (Trin.)

= Leymus angustus (Trin.) Pilger

Leymus chinensis (Trin.) Tzvel.

= Elymus pseudoagropyron (Trin. ex Griseb.) Turcz.

Leymus cinereus (Scribn. \& Merr.) A. Love

$=$ Elymus cinereus Scribn. \& Merr. ${ }^{\mathrm{b}}$

Leymus condensatus (J. Presl) A. Love

= Elymus condensatus Presl.

Leymus flavescens (Scribn. \& J.G. Sm.) Pilger

$=$ Elymus arenicola Scribn. $\&$ Smith

Leymus innovatus (Beal) Pilger

$=$ as Elymus innovatus Bea

Leymus mollis (Trin) Hara ssp. mollis

= Elymus mollis Trin

Leymus paboanus (Claus) Pilg.

= Elymus salsuginosus (Griseb.) Turcz. ex Steud.

Leymus racemosus (Lam.) Tsvelev

$=$ Elymus giganteus $\mathrm{Vahl}$
ND (BPI)

$\operatorname{MT}(99,136,163)$

CA (WSP, 139); CO (OSC, 136, 147); ID (BPI, WSP, 136); MT (WSP, 44, 133, 136); NV (UC); ND (136); OR (BPI, OSC, 44, 135, 136); UT (NY, 136, 147); WA (WSP, 42, 44, 136); WY (BPI, OSC, UC, WIS, 44, 136) CA (BPI, UC); CO (RM); IN (105); IA (ICS, 52, 157); MI (MSC); MT (BPI, 24, 90, 99); OR (NY); UT (BPI); WY (BPI, NY, RM, 50); general (9) OR (BPI, OSC, 135, 136)

WA (WSP, 141)

AK (BPI, WIS, WSP, 22, 136, 137); OR (BPI, 135, 136); WA (CUP, 1); general (9).

ND $(44,136)$

DC (BPI); IA (ICS, 52, 157); MO (109), ND (136); WA (44, 136) 
Table 3. (Continued from previous page)

\begin{tabular}{|c|c|}
\hline Host & Source \\
\hline $\begin{array}{l}\text { Leymus triticoides (Buckl.) Pilger } \\
=\text { Elymus triticoides Buckl. }\end{array}$ & $\begin{array}{l}\text { CA (BPI, 44, 136); CO (NEB); IN (105); NV (BPI, 136, 154); OR (BPI, } \\
\text { OSC, WSP, 135, 136); WA }(44,136) \text {; general (9). }\end{array}$ \\
\hline $\begin{array}{l}\text { Pascopyrum smithii (Rydb.) A. Love } \\
=\text { Agropyron smithii } \mathrm{Rydb} \text {. } \\
=\text { Agropyron occidentale (Scribn.) Scribn }\end{array}$ & $\begin{array}{l}\text { AZ (136); CO (BPI, WSP, 136); DC (BPI); IN (105); KS (BPI, NY, OSC); } \\
\text { IA (BPI, NY, 52, 53, 107, 136, 157, 158); KY (73); MA (NY); MI (71, } \\
\text { 136); MN (45, 75, 85, 127, 136); MO (136); MT (BPI, OSC, WSP, 44, } 99 \text {, } \\
\text { 136); NE (BPI, NEB, 1, 136); NM (BPI, 136, 150); ND (BPI, MSC, 19, } \\
\text { 99, 136, 162); OK (BPI, 23, 41, 97, 125, 136); SD (BPI, FH, OSC, 99, } \\
\text { 106, 136, 156); TX (BPI, 23, 136); UT (FH); WA (WSP, 42, 136); WI } \\
\text { (BPI, UC, 5, 45, 55, 61, 136); WY (BPI, WSP, 14, 44, 50, 136) }\end{array}$ \\
\hline $\begin{array}{l}\text { Psathyrostachys juncea (Fisch.) Nevski } \\
=\text { Elymus junceus Fisch. }\end{array}$ & MT $(44,136)$; ND (WSP, 44, 136); WA (WSP, 136, 147); general (9) \\
\hline $\begin{array}{l}\text { Pseudoroegneria spicata (Pursh) A. Löve } \\
=\text { Agropyron spicatum (Pursh) Schribn. \& Sm. } \\
=\text { Agropyron divergens (Nees ex Steud.) Vasey } \\
=\text { Agropyron inerme (Scribn. \& Smith) Rydb. }\end{array}$ & $\begin{array}{l}\text { CO (BPI); ID (136, 147); IN (105); IA (ICS, 52, 116, 136, 157); MT (BPI, } \\
\text { WSP, 116, 24, 44, 99, 136); NE (NEB); ND (136, 148); OR (WSP, 44, } \\
\text { 136); UT (BPI, 136); WA (WSP, 44, 136); WY (136); northern great } \\
\text { plains and pacific northwest (9) }\end{array}$ \\
\hline Secale cereale $\mathrm{L}$. & 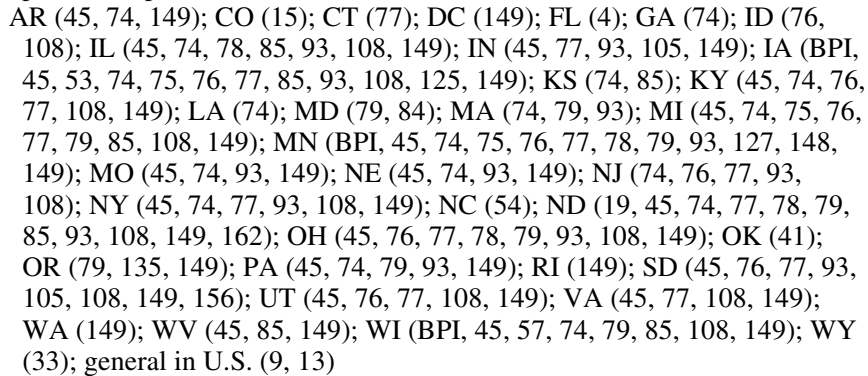 \\
\hline Triticum aestivum L. and T. durum Desf. & $\begin{array}{l}\text { AZ (45, 93, 136); DC (BPI); ID (79); IL (BPI, 45, 74, 78, 85, 108, 149); IN } \\
\text { (BPI, 78, 79, 85, 93, 105); IA (53, 78, 125, 136); KS }(136) ; \mathrm{KY}(45,78) ; \\
\text { ME (BPI); MI (78, 79, 108); MN }(45,74,75,76,77,78,85,93,108,127, \\
\text { 136, 149); MO (136); MT (BPI); NE (45, 74, 76, 78, 149); NY (75, 78, } \\
\text { 136); NC (149); ND (45, 74, 75, 76, 77, 78, 79, 85, 93, 108, 136, 149, } \\
\text { 162); OH (78, 108, 136); OK (41, 125, 136); OR (135, 136); PA (78, 136); } \\
\text { SD (76, 78); UT (45); VA (77, 149); WA (BPI); WV (78); WI (BPI, 45, } \\
\text { 61, 74, 78, 85, 93, 136, 149); WY (33) }\end{array}$ \\
\hline
\end{tabular}

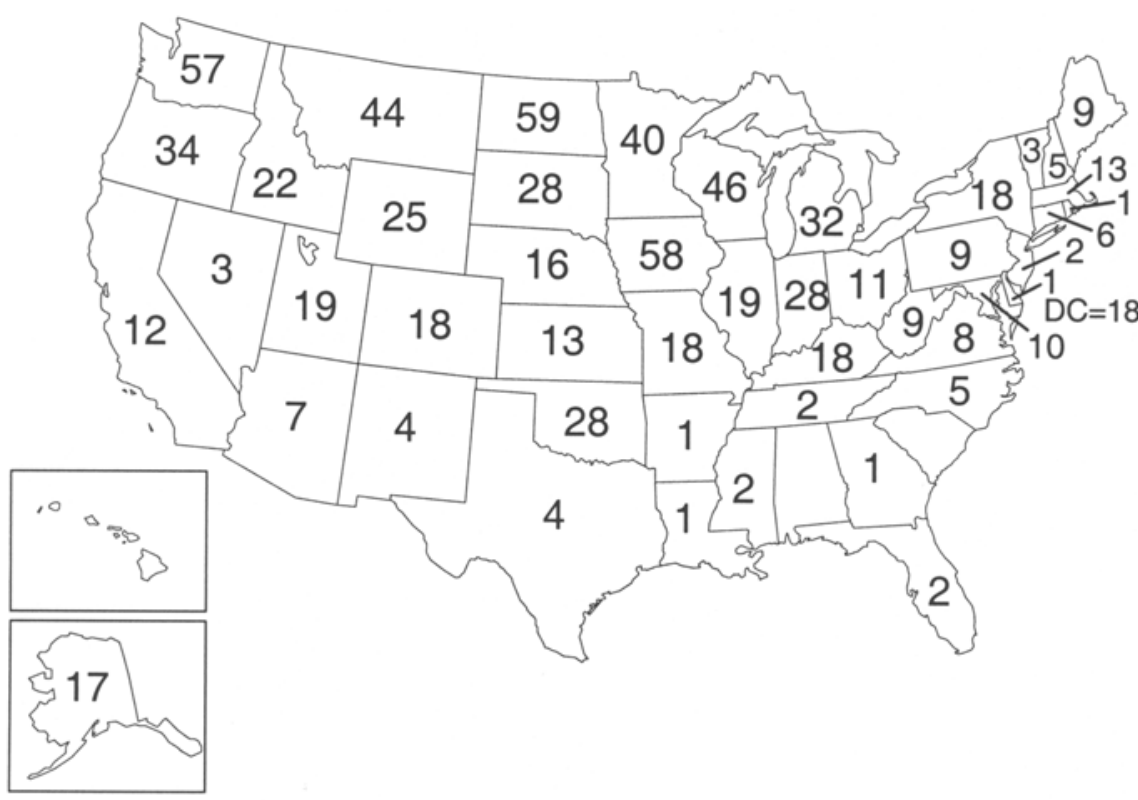

Fig. 2. Number of grass species reported within each state as a host of Claviceps purpurea var. purpurea.

C. grohii is rare in the United States but has been reported from MI, OR, and WA (Table 1). C. grohii was originally described from Carex in Canada (68).

C. purpurea var. purpurea was reported on Cynodon $(25,26)$. However, Porter et al. (122) suggest that a Claviceps responsible for toxin production in Cynodon dactylon
Cynodon spp. (95). Additional studies are needed to determine if Cynodon spp. serve as hosts to $C$. purpurea var. purpurea in nature. Other species or varieties of Claviceps likely are present in the United States, especially on grasses in the Panicoideae. Pammel and Weems (116) found yellow capitula on germinated sclerotia of Claviceps from a Poa species in Iowa. Yellow capitula are not known to occur in C. purpurea var. purpurea.

The taxonomy of grass species is currently undergoing major change. Criteria for separation of genera or species are expanding beyond morphological features or structures, especially in relation to the spikelet, and now include aspects of genetics, physiology, microanatomy, and development. It is likely that present taxonomic relationships and nomenclature of the grasses will continue to change. Therefore, as a base of reference, the names and authorities of grasses as originally listed were retained as synonyms in Tables 1 and 3, and cited in Table 2.

Collections of specimens and hosts of $C$. purpurea in herbaria were helpful in evaluating the host reports of Claviceps species in the United States. The relatively stable attribute of conidial size proved useful in identifying collections with questionable affinity to $C$. purpurea. Additional studies concerning identification of Claviceps spp. should be conducted with fresh collections 
and should focus on features of ascostromata; chemical attributes such as alkaloids present; physical attributes such as sclerotial density, texture, and color; and molecular analyses, including ITS, $\beta$-tubulin, or other sequence determination.

\section{LITERATURE CITED}

1. Abou-Chaar, C. I., Gröger, D., Brady, L. R., and Tyler, V. E. 1961. Interactions of ergot strains in saprophytic culture. Lloydia 24:159-167.

2. Alderman, S. C. 1991. Assessment of ergot and blind seed diseases of grasses in the Willamette Valley of Oregon. Plant Dis. 75:1038-1041.

3. Alderman, S. C. 2003. Diversity and speciation in Claviceps. Pages 195-245 in: Clavicipitalean Fungi: Evolutionary Biology, Chemistry, Biocontrol, and Cultural Impacts. Marcel Dekker, Inc., New York.

4. Alfieri, S. A., Jr., Langdon, K. R., Wehlburg, C., and Kimbrough, J. W. 1984. Index of plant diseases in Florida. Florida Dep. Agric. Consumer Serv., Div. Plant Industry, Bull. No. 11.

5. Allison, J. L., and Chamberlain, D. W. 1942. Grass Diseases in Wisconsin in 1941. Plant Dis. Rep. 26:19-22.

6. Anderson, W. F. 1889. Brief notes on a few common fungi of Montana. J. Mycol. 5:3032.

7. Anderson, F. W. 1889. Supplementary notes. J. Mycol. 5:82-84.

8. Anderson, J. P. 1913. A partial list of parasitic fungi of Decatur County, Iowa. Iowa Acad. Sci. 20:115-131.

9. Anonymous. 1960. Index of Plant Diseases in the United States. U.S. Dep. Agric. Handb. 165. Washington, DC

10. Arnow, L. A. 1994. Koeleria macrantha and $K$. pyramidata (Poaceae): Nomenclatural problems and biological distinctions. Syst. Bot. 19:6-20.

11. Bandyopadhyay, R., Frederickson, D. E., McLaren, N. W., Odvody, G. N., and Ryley, M. J. 1998. Ergot: A new disease threat to sorghum in the Americas and Australia. Plant Dis. 82:356-367.

12. Bellue, M. K. 1954. Ergot on pasture grasses in relation to convulsive ergotism in livestock. Calif. Dep. Agric. Bull. 43:9-11.

13. Bierlein, D. L. 1962. The occurrence of ergot on smooth bromegrass in Michigan. Plant Dis. Rep. 46:838.

14. Bodine, E. W. 1944. Ergot on grasses in Wyoming. Plant Dis. Rep. 28:1106.

15. Bodine, E. W. 1944. Summary of plant diseases in Colorado for 1943. Plant Dis. Rep. (Suppl.) 149:344-351.

16. Boewe, G. H. 1964. Some plant diseases new to Illinois. Plant Dis. Rep. 48:866-870.

17. Boyle, L. W. 1944. Plant disease survey in Washington, 1943. Plant Dis. Rep. (Suppl.) 149:396-400

18. Brady, L. R. 1962. Phylogenetic distribution of parasitism by Claviceps species. Lloydia 25:1-36.

19. Brenckle, J. F. 1917. North Dakota Fungi - I. Mycol. 9:275-293.

20. Brown, H. B. 1916. Life history and poisonous properties of Claviceps paspali. J. Agric. Res. 7:401-406

21. Burton, G. W. 1943. Factors influencing seed setting in several southern grasses. J. Am. Soc. Agron. 35:465-474.

22. Cash, E. K. 1953. A check list of Alaskan fungi. Plant Dis. Rep. (Suppl.) 219.

23. Chester, K. S., and Lefebvre, C. L. 1942. Ergot epiphytotics in southwestern pastures. Plant Dis. Rep. 26:408-410.

24. Chestnut, V. K., and Wilcox, E. V. 1901. The stock-poisioning plants of Montana, a preliminary report. USDA Div. Bot. Bull. No. 26.

25. Conway, K. E., and Taliaferro, C. M. 1990. Occurrence of Claviceps purpurea and hyperparasites on Bermuda grass (Cynodon dactylon) and old world bluestem (Bothriochloa spp.) (Abstr.) Phytopathology 80:1043.

26. Conway, K. E., Taliaferro, C. M., and Shelby, R. A. 1992. First report on formation of sclerotia of Claviceps purpurea on Bermudagrass (Cynodon dactylon). Plant Dis. 76:1077.

27. Criag, J., and Hignight, K. W. 1991. Control of ergot in buffelgrass with triadimefon. Plant Dis. 75:627-629.

28. Cunfer, B. M., and Seckinger, A. 1977. Survival of Claviceps purpurea and C. paspali sclerotia. Mycologia 69:1142-1148.

29. Dale, J. L. 1963. An ergot fungus on Panicum anceps. Plant Dis. Rep. 47:190-191.

30. Dana, B. F., Wolf, S. E., and Powers, L. 1930. Texas fungi. Plant Dis. Rep. 14:240245

31. Davis, J. J. 1924. Notes on parasitic fungi in Wisconsin X. Trans. Wisc. Acad. Sci. 21:271-286

32. Davis, J. J. 1929. Notes on parasitic fungi in Wisconsin XVIII. Trans. Wisc. Acad. Sci. 24:295-301.

33. Davison, A. D. 1958. Plant diseases of economic crops occurring in Wyoming during 1958. Plant Dis. Rep. 42:1409-1410.

34. Duncan, R. A., Jr., Sullivan, R., Alderman, S. C., Spatafora, J. W., and White, J. F., Jr. 2002. Claviceps purpurea var. spartinae var. nov.: An ergot adapted to the aquatic environment. Mycotaxon 81:11-25.

35. Eleuterius, L. N. 1970. Observations on Claviceps purpurea on Spartina alterniflora in the coastal marshes of Mississippi. Gulf Res. Rep. 3:105-109.

36. Eleuterius, L. N., and Meyers, S. P. 1974. Claviceps purpurea on Spartina in coastal marshes. Mycologia 66:978-986.

37. Ellett, C. W. 1957. Diseases not previously reported in Ohio. Plant Dis. Rep. 41:369371

38. Elliott, E. S. 1954. Notes on forage plant diseases observed in northern West Virginia during 1953. Plant Dis. Rep. 38:279-281.

39. Elliott, E. S. 1955. Forage plant diseases observed in West Virginia during 1954. Plant Dis. Rep. 39:318-321.

40. Farr, D. F., Bills, G. F., Chamuris, G. P., and Rossman, A. Y. 1989. Fungi on Plants and Plant Products in the United States. American Phytopathological Society, St. Paul, MN.

41. Featherly, H. I. 1940. Ergot. Oklahoma Agric. Exp. Stn. Mimeogr. Circ. No. 55.

42. Fischer, G. W. 1940. Grass diseases occurring in the Pullman nursery unit of the soil conservation nurseries, Pullman, Washington, during 1939. Plant Dis. Rep. 24:108114

43. Fischer, G. W. 1940. Grass diseases in the Pacific Northwest in 1940. Plant Dis. Rep. 24:481-497.

44. Fischer, G. W., Sprague, R., Johnson, H. W., and Hardison, J. R. 1942. Host and pathogen indicies to the diseases observed on grasses in certain western states during 1941. Plant Dis. Rep. (Suppl.) 137:87-144.

45. Fromme, F. D. 1921. Diseases of cereal and forage crops in the United States in 1920. Plant Dis. Bull. (Suppl.) 15:116-176.

46. Fyles, F. 1915. A preliminary study of ergot of wild rice. Phytopathology 5:186-192.

47. Gessner, R. V., and Goos, R. D. 1973. Fungi from Spartina alterniflora in Rhode Island. Mycologia 65:1296-1301.

48. Gessner, R. V. 1977. Seasonal occurrence and distribution of fungi associated with
Spartina alterniflora from a Rhode Island estuary. Mycologia 69:477-491.

49. Gessner, R. V. 1978. Spartina alterniflora seed fungi. Can. J. Bot. 56:2942-2947.

50. Gilbertson, R. L., Cummins, G. B., and Darnall, E. D. 1979. Indexes to W. G. Solheim's mycoflora saximontanesis exsiccata. Mycotaxon 10:49-92.

51. Gilman, J. C. 1932. First supplementary list of fungi from Iowa. Iowa State Coll. J. Sci. 6:357-365.

52. Gilman, J. C. 1949. Second supplementary list of parasitic fungi from Iowa. Iowa State Coll. J. Sci. 23:261-272.

53. Gilman, J. C., and Archer, W. A. 1929. The fungi of Iowa parasitic on plants. Iowa State Coll. J. Sci. 3:299-507.

54. Grand, L. F. 1985. North Carolina plant disease index. N.C. Agric. Res. Serv. Tech Bull. 240 (revised).

55. Greene, H. C. 1942. Notes on Parasitic Fungi II. Trans. Wisc. Acad. Sci. 34:83-98.

56. Greene, H. C. 1947. Notes on Wisconsin parasitic fungi IX. Trans. Wisc. Acad. Sci. 38:235-248.

57. Greene, H. C. 1948. Notes on Wisconsin parasitic fungi. X. Am. Midl. Naturalist 39:444-456.

58. Greene, H. C. 1949. Notes on Wisconsin parasitic fungi. XII. Am. Midl. Naturalist 41:726-739.

59. Greene, H. C. 1949. Notes on Wisconsin parasitic fungi. XIII. Am. Midl. Naturalist 41:740-758

60. Greene, H. C. 1949. Fungi of the Wisconsin arboretum. Trans. Wisc. Acad. Sci. 39:47-82.

61. Greene, H. C. 1951. Host index of parasitic fungi collected on plants in Wisconsin, 1880-1950. Edwards Bros., Ann Arbor, MI.

62. Greene, H. C. 1952. Notes on Wisconsin parasitic fungi. XV. Am. Midl. Naturalist 48:37-54

63. Greene, H. C. 1952. Notes on Wisconsin parasitic fungi. XVI. Am. Midl. Naturalist 48:741-757.

64. Greene, H. C. 1953. Notes on Wisconsin parasitic fungi XVIII. Trans. Wisc. Acad. Sci. 42:69-81

65. Greene, H. C. 1953. Notes on Wisconsin parasitic fungi. XIX. Am. Midl. Naturalist 50:501-508

66. Greene, H. C. 1956. Notes on Wisconsin parasitic fungi XXI. Trans. Wisc. Acad. Sci. 44:29-43.

67. Griffiths, D. 1901. Contributions to a better knowledge of the Pyrenomycetes. Bull. Torrey Bot. Club. 28:236-241

68. Groves, J. W. 1943. A new species of Claviceps on Carex. Mycologia 35:604-609.

69. Guba, E. F. 1939. List of second hundred fungi of Nantucket. Rhodora 41:508-520.

70. Hardison, J. R. 1941. Grass diseases in Michigan in 1940. Plant Dis. Rep. 25:120125

71. Hardison, J. R. 1942. Grass diseases in Michigan in 1941. Plant Dis. Rep. 26:67-75.

72. Hardison, J. R. 1943. Grass diseases in Kentucky in 1942. Plant Dis. Rep. 27:103108

73. Hardison, J. R. 1945. Observations on grass diseases in Kentucky, September, 1942 to Spetember, 1944 and a preliminary check list. Plant Dis. Rep. 29:76-85.

74. Haskell, R. J. 1919. Summary of plant diseases in the United States in 1918 IV. Diseases of cereal and forage crops. Plant Dis. Bull. (Suppl.) 4:119-159.

75. Haskell, R. J. 1924. Diseases of cereal and forage crops in the United States in 1923. Plant Dis. Rep. (Suppl.) 35:244-317.

76. Haskell, R. J. 1926. Diseases of cereal and forage crops in the United States in 1925. Plant Dis. Rep. (Suppl.) 48:301-381. 
77. Haskell, R. J. 1928. Diseases of cereal and forage crops in the United States in 1927. Plant Dis. Rep. (Suppl.) 62:302-353.

78. Haskell, R. J., and Wood, J. I. 1923. Diseases of cereal and forage crops in the United States in 1922. Plant Dis. Bull. (Suppl.) 27:164-266.

79. Haskell, R. J., and Wood, J. I. 1929. Diseases of cereal and forage crops in the United States in 1928. Plant Dis. Rep. (Suppl.) 71:259-323.

80. Hoerner, G. R. 1938. The fungus flora of the Peavy arboretum. Plant Dis. Rep. 22:264267.

81. Holmgren, P. K., Holmgren, N. H., and Barnett, L. C., eds. 1990. Index Herbariorum: Part I: The Herbaria of the World. 8th ed. New York Botanical Garden, Bronx, NY.

82. Honey, E. E., and Vaughan, R. E. 1944. Plant diseases observed in Wisconsin in 1943. Plant Dis. Rep. (Suppl.) 147:199-209.

83. Isakeit, T., Odvody, G. N., and Shelby, R. A. 1998. First report of sorghum ergot caused by Claviceps africana in the United States. Plant Dis. 82:592.

84. Jeffers, W. F., Cox, C. E., Jehle, R. A., Weaver, L. O., Morgan, O. D., and Moore, J. E. 1951. Notes on occurrence of some plant diseases in Maryland during 1950. Plant Dis. Rep. 35:385-386.

85. Johnson, A. G., and Haskell, R. J. 1920. Diseases of cereal and forage crops in the United States in 1919. Plant Dis. Bull. (Suppl.) 8.

86. Jones, D. D., and Tyler, V. E. 1955. The pharmacognosy of smooth brome ergot. J. Am. Pharm. Assoc. 44:480-483.

87. Kartesz, J. T. 1994. A synonymized checklist of the vascular flora of the United States, Canada, and Greenland, 2nd ed. Timber Press, Portland, OR.

88. Kawatani, T. 1953. Hosts of Claviceps. Bull. Natl. Hyg. Lab. 70:127-152.

89. Kellerman, W. A., and Carleton, M. A. 1887. Second list of Kansas parasitic fungi, together with their host-plants. Trans. Kansas Acad. Sci. 10:88-99.

90. Kelsey, F. D. 1889. Notes on the fungi of Helena, Mont. J. Mycol. 5:80-82.

91. Kernkamp, M. F., Kroll, R., and Woodruff, W. C. 1976. Diseases of cultivated wild rice in Minnesota. Plant Dis. Rep. 60:771-775.

92. Kilpatrick, R. A. 1956. Diseases of forage crops in New England and New York in 1956. Plant Dis. Rep. 40:1054-1057.

93. Kirby, R. S., and Archer, W. A. 1927. Diseases of cereal and forage crops in 1926. Plant Dis. Rep. (Suppl.) 53:111-208.

94. Lambert, D. H., and McIlveen, W. D. 1976. Acylomus sp. infesting ergot sclerotia. Ann. Entomol. Soc. Am. 69:34

95. Langdon, R. F. N. 1954. New species of Claviceps. Univ. Queensl. Pap. 3:39-40.

96. Langdon R. F. N. 1954. The origin and differentation of Claviceps species. Univ. Queensl. Pap. 3:61-68.

97. Larsh, H. W. 1944. Summary of plant diseases in Oklahoma, 1943. Plant Dis. Rep. (Suppl.) 149:317-326

98. Lefebvre, C. L. 1939. Ergot of Paspalum. Phytopathology 29:35-367.

99. Lefebvre, C. L., and Johnson, H. W. 1941. Collections of fungi, bacteria, and nematodes of grasses. Plant Dis. Rep. 25:556-579.

100. Loveless, A. R. 1964. Use of the honeydew state in the identification of ergot species. Trans. Br. Mycol. Soc. 47:205-213.

101. Loveless, A. R. 1971. Conidial evidence for host restriction in Claviceps purpurea. Trans. Br. Mycol. Soc. 56:419-434.

102. Loveless, A. R., and Herd, G. W. 1964. A redescription of Claviceps sulcata Langdon. Kirkia 4:41-44.
103. Loveless, A. R., and Peach, J. M. 1974. Evidence for the genotypic control of spore size in Claviceps purpurea. Trans. Br. Mycol. Soc. 63:612-616.

104. Mackie, W. W., and Snyder, W. C. 1945. Ergot of dallis grass in California. Plant Dis. Rep. 29:85.

105. Mains, E. B. 1925. Observations concerning the disease susceptibility of cereals and wild grasses. Proc. Indiana Acad. Sci. 34:289295.

106. Mankin, C. J. 1969. Diseases of grasses and cereals in South Dakota. S.D. Agric. Exp. Stn. Tech. Bull. 35.

107. McNew, G. L., Johnson, J. I., and Wilsie, C. P. 1948. A study of bromegrass diseases and their control. In: Report on Agricultural Research for the year ending June 30, 1948. Iowa Agric. Exp. Stn.

108. Melchers, L. E. 1925. Diseases of cereal and forage crops in the United States in 1924. Plant Dis. Rep. (Suppl.) 40:106-191.

109. Mihail, J. D. 1993. Diseases of alternative crops in Missouri. Can. J. Plant Pathol. 15:119-122.

110. Miles, L. E. 1935. The ascomycetes of Mississippi. Plant Dis. Rep. 19:39-66.

111. Mitchell, D. T., and Cooke, R. C. 1968. Some effects of temperature on germination and longevity of sclerotia in Claviceps purpurea. Trans. Br. Mycol. Soc. 51:721-729.

112. Möller, A. 1901. Phycomyceten und Ascomyceten. Untersuchungen aus Brasilien. Gustav Fischer, Jena.

113. Munkvold, G. P., Carson, T., and Thoreson, D. 1997. Outbreak of ergot (Claviceps purpurea) in Iowa barley, 1996. Plant Dis. 81:830.

114. Norton, J. B. S. 1902. Plant diseases of Maryland in 1902. Rep. Maryland State Hortic. Soc. 5:90-99.

115. Pammel, L. H. 1891. Some fungus diseases of Iowa forage plants. Iowa Acad. Sci. 1:9394.

116. Pammel, L. H., and Weems, J. B. 1900 Grasses of Iowa. Iowa Agric. Coll. Exp. Stn. 54:71-621.

117. Pantidou, M. E. 1959. Claviceps from Zizania. Can. J. Bot. 37:1233-1236.

118. Parris, G. K. 1959. A revised host index of Mississippi Plant Diseases. Miss. State Univ. Bot. Dep. Misc. Publ. 1.

119. Pazoutova, S., Olsovska, J., Linka, M., Kolinska, R., and Flieger, M. 2000. Chemoraces and habitat specialization of Claviceps purpurea populations. Appl. Environ. Microbiol. 66:5419-5425.

120. Pazoutova, S. 2001. The phylogeny and evolution of the genus Claviceps. Mycol. Res. 105:275-283.

121. Pierstorff, A. L. 1937. Plant diseases reported in Ohio. Plant Dis Rep. 21:274-275.

122. Porter, J. K., Bacon, C. W., and Robbins, J. D. 1974. Major alkaloids of a Claviceps Isolated from Toxic Bermuda grass. J. Agric. Food. Chem. 22:838-841

123. Povah, A. H. 1931. Fungi of Rover Rock Michigan. Pap. Mich. Acad. Sci. 13:173-189.

124. Presley, J. T. 1947. A host index of Mississippi plant diseases. Plant Dis. Rep. (Suppl.) 169:55-160.

125. Preston, D. A. 1945. Host index of Oklahoma plant diseases. Oklahoma Agric. Exp. Stn. Tech. Bull. 21.

126. Preston, D. A. 1947. Host index of Oklahoma Plant Diseases, Supplement, 1947. Oklahoma Agric. Exp. Stn. Tech. Bull. T-21 (Suppl.).

127. Preston, D. A., and Dosdall, L. 1955. Minnesota plant diseases. USDA-ARS Plant Dis. Epidemics Identification Sect. Spec. Publ. No. 8

128. Raabe, R. D., Conners, I. L., and Martinez,
A. P. 1981. Checklist of plant diseases in Hawaii. College of Tropical Agriculture and Human Resources, University of Hawaii. Information Test Ser. No. 22. Hawaii Inst. Trop. Agric. Human Resources.

129. Ray, W. W. 1954. Unusual or new occurrences of fungus pathogens on grasses in Nebraska. Plant Dis. Rep. 38:583-587.

130. Roane, C. W., and Roane, M. K. 1994. Graminicolous fungi of Virginia: Fungi associated with cereals. Virginia J. Sci. 45:279296.

131. Rogerson, C. T. 1956. Diseases of grasses in Kansas: 1953-55. Plant Dis. Rep. 40:388397.

132. Rosen, H. R. 1920. Ergot on Paspalum. Mycologia 12:40-41.

133. Shaw, C. G. 1973. Host fungus index for the Pacific Northwest - I. Hosts. Wash. State. Agric. Exp. Stn. Bull. 765:1-121.

134. Sprague, R. 1942. Miscellaneous notes: Mostly North Dakota. Plant Dis. Rep. 26:327-328

135. Sprague, R. 1942. A revised check list of parasitic fungi on cereals and other grasses in Oregon. Plant Dis. Rep. (Suppl.) 134.

136. Sprague, R. 1950. Diseases of Cereals and Grasses in North America. The Ronald Press Co., New York.

137. Sprague, R. 1955. Check list of the fungi of the Glacier Bay National Monument, Alaska. Res. Stud. State Coll. Wash. 23:202-224.

138. Sprague, R. 1957. Some fungi on western gramineae XI. Mycologia 49:837-853.

139. Sprague, R. 1958. Some leafspot fungi on western gramineae - XII. Mycologia 50:814830

140. Sprague, R. 1960. Some leafspot fungi on western gramineae - XIII. Mycologia 52:357377.

141. Sprague, R. 1960. Some leafspot fungi on western gramineae - XIV. Mycologia 52:698718.

142. Sprague, R. 1962. Some leafspot fungi on wesern gramineae - XV. Mycologia 54:44 61.

143. Sprague, R. 1962. Some leafspot fungi on western gramineae - XVI. Mycologia 54:593610

144. Sprague, R. 1962. Some fungi on western species of Cyperaceae. II. Res. Stud. State Coll. Wash. 30:45-63.

145. Sprague, R., Fischer, G. W., and Meiners, J. P. 1948. Some new disease records of Gramineae in the western United States. Plant Dis. Rep. 32:98-114.

146. Sprague, R., and Meiners, J. P. 1948. Additional parasitic fungi on Gramineae in the Inland Empire. Plant Dis. Rep. 32:245-247.

147. Sprague, R., and Meiners, J. P. 1949. Some new disease records of Gramineae in the western United States - II. Plant Dis. Rep. 33:147-158

148. Sprague, R., and Meiners, J. P. 1949. Some new disease records of Gramineae in the western United States - III. Plant Dis. Rep. 33:259-270.

149. Stakman, E. C. 1922. Diseases of cereal and forage crops in the United States in 1921. Plant Dis. Bull. (Suppl.) 21:139-254

150. Standley, P. C. 1916. Fungi of New Mexico. Mycologia 8:142-177.

151. Starr, G. H. 1937. Notes on plant diseases in Wyoming. Plant Dis. Rep. 21:328-330.

152. Steinmetz, F. H., and Wright, C. M. 1943. A study of Claviceps on Zizanea. Phytopathology $33: 12-13$

153. Stevens, F. L., and Hall, J. G. 1910. Three interesting species of Claviceps. Bot. Gazette 50:460-463.

154. Stevenson, J. A., and Archer, W. A. 1940. A contribution to the fungus flora of Nevada. Plant Dis. Rep. 24:93-103. 
155. Tervet, I. W. 1944. Minnesota. Plant disease survey in Minnesota. Plant Dis. Rep. (Suppl.) 147:210-217.

156. Tervet, I. W. 1944. Diseases in South Dakota in 1943. Plant Dis. Rep. (Suppl.) 149:308311.

157. Tiffany, L. H. 1948. Further observations on Claviceps purpurea. Proc. Iowa Acad. Sci. 55:217-221.

158. Tiffany, L. H., Shearer, J. F., and Knaphus, G. 1990. Parasitic fungi of four tallgrass prairies of northern Iowa: Distribution and prevalence. JIAS 97:157-166.
159. Trelase, W. 1882. Preliminary list of Wisconsin parasitic fungi. Trans. Wisc. Acad. Sci. 6:106-144.

160. Velasquez-Valle, R., Odvody, G., Isakeit, T., and Williams, H. 1998. Spread of sorghum ergot in the U.S.A. and Mexico. Phytopathology (Suppl.) 88:S123.

161. Watson, L., and Dallwitz, M. J. 1992. The grass genera of the world. CAB International, University Press, Cambridge.

162. Weniger, W. 1924. North Dakota Agricultural Experiment Station Bulletin 176.

163. Whetzel, H. H., and Reddick, D. 1911. A method of developing Claviceps. Phytopathology 1:50-52.

164. Williams, T. A. 1892. Some plants injurious to stock. S.D. Agric. Coll. Exp. Stn. Bull. 31:21-44.

165. Wilson, G. W. 1910. Preliminary list of the parasitic fungi of Fayette County, Iowa. Iowa Acad. Sci. 17:47-79.

166. Zumo, N., Gourley, L. M., Trevathan, L. E., and Gonzalez, M. S. 1998. Occurrence of ergot (sugary disease) incited by a Sphacelia sp. on sorghum in Mississippi in 1997. Plant Dis. 82:590 